\title{
MedienPädagogik
}

www. medienpaed.com

Themenheft Nr. 23: Visuelle Kompetenz

\section{Aussteigen aus dem Rechtsextremismus Foto-Praxis, bildwissenschaftliche Analyse und Ausstellungsarbeit als Methoden individueller Reflexion und des wissenschaftlichen und (sozial)pädagogischen Kompetenzerwerbs}

Ulrike Pilarczyk und Fabian Wichmann

\begin{abstract}
Der Beitrag zeigt die medienpädagogischen und bildwissenschaftlichen Dimensionen eines zeitlich und thematisch gestaffelten Projektes auf. Ausgangspunkt war das in den Jahren 2009/10 von der Organisation EXIT-Deutschland in Berlin mit Aussteigern(-innen) aus der rechtsextremen Szene initiierte Fotoprojekt «Lebensbilder». Die im Rahmen biografisch orientierter, medien- und sozialpädagogischer Fallarbeit entstandenen Fotos wurden als Prozess individueller Bilderzeugung und als Mittel zur Reflexion der jeweiligen Lebenssituation der Aussteiger/innen verstanden und eingesetzt. Eine Ausstellung schloss diese erste Phase des hier beschriebenen Projektes ab.

Dem Wunsch folgend, die praktischen Erfahrungen aus der Arbeit mit Fotos wissenschaftlich überprüfen zu lassen, wurden in den Jahren 2011 und 2012 mit Studierenden am erziehungswissenschaftlichen Institut der TU Braunschweig die "Lebensbilder» zunächst einer wissenschaftlichen Bildanalyse unterzogen und anschliessend in einem gestalterisch-interpretativen Projekt durch die Studierenden zu einer Ausstellung weiterentwickelt.
\end{abstract}

1 Fotografieren als Bildproduktions- und Reflexionsprozess: Das Fotoprojekt «Lebensbilder» 2010 (EXIT-Deutschland, Berlin; Fabian Wichmann)

Mit acht Aussteiger/innen aus der rechtsextremen Szene initiierten Mitarbeiter von EXIT-Deutschland im Winter/Frühjahr 2009/2010 das Fotoprojekt "Lebensbilder». ${ }^{1}$ Drei von ihnen befanden sich zu dieser Zeit im akuten Ausstiegsprozess, bei den anderen lag dieser mehr als zwei Jahre zurück. Sie waren in unterschiedlichen subkulturellen Gruppen aktiv gewesen oder mit diesen assoziiert (Freie Kameradschaften, Autonome Nationalisten, völkische Zusammenschlüsse oder SkinheadKameradschaften), z. T. auch als Führungskader und Kameradschaftsführer/innen. ${ }^{2}$

1 Das Fotoprojekt «Lebensbilder» war Teil der Werkstattarbeit von EXIT-Deutschland und wurde im Rahmen des EXIT-Projektes Seitenwechsel - Ausstieg als Einstieg in ein neues Leben durch das Bundesprogramm XENOS - Ausstieg als Einstieg gefördert.

2 U.a. in der Freiheitlichen Deutschen Arbeiterpartei (FAP), einer Kleinpartei, die von 1979 bis 1995 bestand und 1995 verboten wurde; in der Hilfsorganisation für nationale politische Gefangene 
Das Projekt «Lebensbilder» war ein Versuch, neue Möglichkeiten zur Gestaltung einer effektiven und nachhaltigen Ausstiegsarbeit zu erschliessen, denn EXITDeutschland ist eine Initiative, die Angehörige politisch extremer Szenen im Prozess des Aussteigens unterstützt. ${ }^{3}$

Diese Unterstützung wird dringend gebraucht, denn der Ausstieg aus der rechtsextremen Szene markiert grundsätzlich einen Wendepunkt im Leben derjenigen, die sich dazu entschliessen - verbunden mit dem Wunsch nach persönlicher Sicherheit, Bildung und Arbeit, sozialer Einbindung sowie der Suche nach einem neuen Weltbild, Sinn und Orientierung. Soll der Ausstieg nachhaltig sein, sind die kritische Reflexion, Aufarbeitung sowie das erfolgreiche Infragestellen und Überwinden der bisherigen handlungs- und richtungsweisenden Ideologie Voraussetzung. ${ }^{4}$ Dafür ist es unabdingbar, bisherige Bezugsgruppen, Parteien, häufig auch das soziale Umfeld zu verlassen - ein Prozess, der den Betroffenen die Mobilisierung aller Ressourcen abverlangt und sie schnell an die Grenzen ihrer individuellen Möglichkeiten bringt. Ein- und Ausstiegsgründe sind, so zeigt die Praxis, individuell differenziert und multikausal (Borstel 2011, Wagner, Borstel u. Wichmann 2010, Möller u. Schumacher 2007, ZDK 2002). Die mit dem Ausstieg verbundene Diskontinuitätserfahrung und die mit der Identitäts-(Re)Konstruktion verbundenen Problemlagen sind, abhängig von der Person, seiner Funktion, der Verweildauer und der ehemaligen Bezugsgruppe, entsprechend komplex (Wouters 2012, ZDK 2002). Im Rahmen des EXIT-Deutschland Case Managements wird auf unterschiedliche Methoden des biografischen Arbeitens zum Verstehen subjektiver Sinnstrukturen und für konkrete Unterstützungsangebote (Jansen 2011) zurückgegriffen.

Eine bildbasierte Methode der Analyse der biografischen Situation und individuellen Befindlichkeit sowie daran anschliessende Erörterungen von Interventionsmöglichkeiten erschienen vor diesem Hintergrund methodisch vielversprechend. Ziel des biografisch-reflexiv angelegten Fotoprojektes «Lebensbilder» war, eine Auseinandersetzung mit der aktuellen Lebenssituation in Gang zu setzen, die durch die Fallbetreuer - über eine Analyse des Materials - durch die Erschliessung konkreter, d. h. lebenspraktischer Interventionsmöglichkeiten und Unterstützungsangebote begleitet werden sollte. Hintergrund dieser Idee war die Erfahrung, die alle (Sozial-)Pädagogen machen, wenn sie in den Bereich des vage Gefühlten, Nichtsag-

und deren Angehörige e. V. (HNG), einer Organisation, die bundesweit rechtsextreme verurteilte Straftäter während und nach ihrer Haftzeit in Justizvollzugsanstalten betreute und unterstützte und 2011 verboten wurde; in der Heimattreuen Deutschen Jugend e. V. (HDJ), einem 1990 gegründeten rechtsextremen deutschen Jugendverband mit neonazistischer Ausrichtung, der 2009 verboten wurde.

3 http://www.exit-deutschland.de/EXIT/EXIT-Deutschland-Ausstiege-aus-dem-RechtsextremismusE1240.htm?RID=301 (download vom 1.3.13). Laut Bundesregierung ist EXIT-Deutschland in Bezug auf die extern nachvollziehbaren Fallzahlen eines der erfolgreichsten Deradikalisierungsprogramme im Bereich Rechtsextremismus. Deutscher Bundestag Drucksache 17/9119.

4 Was heisst für uns Ausstieg? Ausstiegsdefinition von EXIT-Deutschland http://www.exit-deutschland.de/EXIT/Navigation/Ausstieg/Ausstieg-K332.htm (download vom 1.3.13). 
baren geraten, so dass gut gemeinte Unterstützung häufig fehlgeht, wenn sie die Situation vor ihrem eigenen Erfahrungshintergrund nicht angemessen einschätzen können. Erschwerend kommt hinzu, dass für die sozialpädagogische Auseinandersetzung im Sinne einer Einzelfallhilfe mit Personen aus der rechtsextremen Szene - ausgehend von einem Jugendparadigma - zumeist jugendspezifische pädagogische Modelle und Methoden Anwendung finden. Diese werden in ihrer analytischen Tiefe nicht dem Erfahrungshorizont der Zielgruppe von EXIT-Deutschland gerecht, da diese gerade im Ausstiegsprozess häufig älter sind (Borstel 2011). ${ }^{5}$

Die Unterstützung von Ausstiegswilligen bei der Herauslösung aus der rechtsextremen Szene und beim Aufbau eines Lebens jenseits von Ideologie, Gewalt und Aktionismus hat jedoch nicht nur für die Betroffenen selbst positive Auswirkungen. Der Ausstieg von Rechtsextremen ist vielmehr für die strategische Auseinandersetzung mit dem Rechtsextremismus entscheidend, trägt er doch zur Destabilisierung der rechten Szene bei. Neben der präventiven Arbeit ist Ausstiegsarbeit daher für die Eindämmung von Rechtsextremismus von elementarer Bedeutung.

\section{Das Fotografieren als Prozess individueller Bilderzeugung und Reflexion}

Die acht Projekt-Teilnehmer/innen, zu denen sechs Männer zwischen 16 und 36 Jahren und zwei Frauen, 32 und 38 Jahre alt, gehörten, stellten sich freiwillig und unabhängig voneinander der Aufgabe, die eigene Vergangenheit, Gegenwart und Zukunft über das Fotografieren mit einer Einwegkamera ins Bild zu setzen. Aus nachvollziehbaren Gründen sollten keine Personen identifizierbar abgebildet sein. Die im digitalen Zeitalter schon anachronistisch anmutenden analogen Einwegkameras waren hierfür mit Bedacht gewählt. Damit wurde zum einen eine bewusstere Motivsuche angeregt, zum anderen verhindert, dass spontane Löschvorgänge am Display, wie sie digitale Kameras ermöglichen, den Bildproduktionsprozess abrupt beenden. Auf einer eher symbolischen Ebene sollte mit der Wahl der analogen Technik auch der biografische Bezug des Projektes aufgenommen werden, denn die Fotografien der Einwegkamera liessen sich nicht ohne Weiteres löschen oder verändern wie eben auch die Vergangenheit nicht. Wohl kann man die Bilder, die die Fotos zeigen, wie auch die eigene Biografie im Nachhinein aus verschiedenen Perspektiven betrachten und interpretieren.

Das Fotografieren als einen Bilderzeugungsprozess zu begreifen, der Reflexion ermöglicht, setzt allerdings die Annahme voraus, dass auch Fotos nicht professioneller Fotografen weder zufällig entstehen, noch, dass sie lediglich Abbildungen von etwas sind, das sich im Moment der Aufnahme vor dem Objektiv befand. Das Fotografieren muss vielmehr als individueller Bilderzeugungsprozess betrachtet

5 Beim modernen Rechtsextremismus handelt es sich nicht ausschliesslich um ein Jugendphänomen, wenngleich der Einstieg zumeist in der Jugendphase stattfindet, die Hauptadressaten Jugendliche sind und auch seit über 20 Jahren das nachwachsende aktionistische, straftatenrelevante Potenzial bilden. 
werden, über den die Fotografierenden etwas ausdrücken und kommunizieren. Dazu können vorfotografisch Vorstellungen gehören, was man fotografieren will, auf jeden Fall das Wählen eines Ausschnittes und auch bestimmter technischer Mittel: z. B. S/W oder Farbe, Schärfe/Unschärfe, Zoom usw. sowie auch des Standpunktes, von dem aus das Motiv aufgenommen wird. Nachfotografisch gehören zu diesem Prozess auch das Auswählen/Autorisieren der gültigen Bilder und eventuelle Nachbearbeitungen. Gerade die nachfotografischen Auswahlprozesse haben beim Fotografieren ein besonderes Gewicht. Denn da sich der Moment der Belichtung der Einflussnahme weitgehend entzieht, lässt sich auch nie ganz ausschliessen, dass Bildelemente zufällig ins Bild geraten sein und sich damit auch bildbestimmende Strukturen einfach ergeben haben könnten. Man kann wohl ein Bild entwerfen, aber wenn der Auslöser gedrückt wird, übernimmt ein technischer Apparat das Bildermachen. Häufig entstehen dabei Fotos, die man so nicht geplant hatte. Nicht nur in der alltäglichen Fotopraxis wird daher ausgewählt, was zu den ursprünglichen Intentionen passte oder gefällt, auch die professionelle Fotografie aller Anwendungsgebiete wird heute über nachfotografische Auswahlen bestimmt.

Dem fotospezifischen Problem von Zufall und Intention begegnete man im Projekt «Lebensbilder» methodisch durch die Aufforderung an die Teilnehmer/innen des Projektes, nach der Entwicklung fünf bis sieben Aufnahmen auszuwählen, die sie für das Thema und ihre eigene Situation für besonders aussagekräftig erachteten. Dieser Prozess, da projektgebunden initiiert und gesteuert, war nicht nur thematisch fokussiert, sondern auch spezifisch adressiert, denn ihnen war bekannt, dass die Fotografien anschliessend von den Mitarbeitern von EXIT ausgewertet wurden. Der Prozess der Bildproduktion wird darüber hinaus durch innere Bilder, psychische Dispositionen, individuell-biografische und kulturell-gesellschaftliche Erfahrungen mit bestimmt.

Mit der Auswahl der Bilder durch die Teilnehmer/innen im Projekt «Lebensbilder» wurde sichergestellt, dass jene Bilder im Zentrum der Betrachtung standen, die die Fotografen/-innen für sich selbst und das Thema für relevant erachteten. Die Bilder wurden damit nachträglich als bedeutungsvolle Äusserung autorisiert, für die Mitarbeiter von EXIT hatten sie damit auch Mitteilungscharakter. Durch die Aufforderung, zusätzlich Unterschriften zu finden und/oder die Fotografien zu kommentieren, sollte bei den Aussteigern/-innen darüber hinaus ein reflexiver, textförmiger Umgang mit dem Bildmaterial angestossen und methodisch das unvermeidlich Zufällige in einen reflexiv interpretierenden Zusammenhang gebracht werden. 
Das Fotoprojekt «Lebensbilder» endete 2010 mit einer ersten Ausstellung ausgewählter Bilder und Texte beim 15. Deutschen Präventionstag im Mai 2010 in Berlin mit durchweg positiver Resonanz. ${ }^{6}$

Allerdings zeigte sowohl die Auswertung der Fotografien durch die Mitarbeiter von EXIT wie auch die Feedbacks zur Ausstellung, dass die ausdrucksstarke Bildlichkeit und auch die energetische Intensität mancher Fotos die Betrachter/innen zwar stark berührten, dass ihnen die Bilder aber gerade dort, wo sie auf Innenwelten und individuelle Verarbeitungsprozesse verwiesen, rätselhaft blieben. Die hochkomplexen ästhetischen Produkte forderten daher eine bildwissenschaftliche Analyse geradezu heraus.

Auf der Suche nach methodischen Ansätzen zur Analyse der Fotografien schien das Verfahren der seriell-ikonografischen Fotoanalyse (Pilarczyk u. Mietzner 2005) für diesen Bestand als besonders geeignet, da es eine Forschungsstrategie bietet, über die bildhafte formulierte Erfahrungs- und Wissensbestände erschlossen werden können. In qualitativ-empirischen Untersuchungen hatte sich das methodische Verfahren bereits mehrfach bewährt (Pilarczyk 2004, 2006, 2009).

\section{Fotografische Bilder als Quelle erziehungswissenschaftlicher Forschung und Reflexion (Sommersemester 2011, TU-Braunschweig; Ulrike Pilarczyk)}

Die bildanalytische Auswertung der Fotografien aus dem Projekt «Lebensbilder» fand im Sommersemester 2011 am Institut für Erziehungswissenschaft der TUBraunschweig im Rahmen eines Workshops mit Studierenden des Lehramtes und der Erziehungswissenschaften und in Kooperation mit EXIT-Deutschland unter Leitung von Ulrike Pilarczyk und Fabian Wichmann statt. Mit der Analyse verband sich auf der Seite der universitären Ausbildung die Erwartung eines Zugewinns an bildwissenschaftlich methodischer Kompetenz und inhaltlich einer aktiven Auseinandersetzung der Studierenden mit einem drängenden gesellschaftlichen Problem, dem Rechtsextremismus in Deutschland. Auf der Seite von EXIT-Deutschland war man überzeugt, über die methodisch geleitete tiefere Erschliessung der fotografischen Bilder auch eine Erweiterung der Perspektiven für die sozialpädagogische Arbeit im Rahmen des Case Managements zu erlangen. Denn es hatte sich ja im Projekt gezeigt, dass Bilder gerade dann an die Stelle der Rede treten, wenn diese innere Prozesse und Befindlichkeiten noch nicht, nicht mehr oder gar nicht auszudrücken vermag. So sollte eine methodisch gestützte, bildwissenschaftliche Analyse helfen, einen diskursiven Zugang zu den im Bild verschlüsselten Erfahrungen, Wünschen und Botschaften öffnen. Darüber hinaus sollte geprüft werden, ob und wie sich strukturell bildwissenschaftliche Verfahren in die praktische Ausstiegsarbeit von EXIT-Deutschland implementieren lassen.

6 Dazu wurde ein Katalog gestaltet, in dem eine Auswahl der Fotografien mit den dazugehörigen Titeln und Bildkommentaren publiziert wurde (EXIT-Deutschland 2011). 


\section{Das Verfahren der seriell-ikonografischen Fotoanalyse}

Die Forschungsfrage, die die seriell-ikonografischen Fotoanalysen der Studierenden im Seminar leitete, war, ob und wie (Ausstiegs-)Erfahrungen in den visuellen Formulierungen der Aussteiger/innen verarbeitet sind und kommuniziert werden. Dafür arbeiteten die Studierenden zunächst bildanalytisch ausschliesslich mit den Foto-Serien. Methodisch wurde darüber eine Konzentration auf die Bildlichkeit intendiert, da die Erfahrung bei der Auswertung visueller Quellen bislang gezeigt hatte, dass die Versuchung fast unwiderstehlich gross ist, Kontextwissen zu Bildwissen umzudeuten. Kommentare und Bildtitel der Fotografen/-innen wurden erst später in die Analyse mit einbezogen. Informationen zu Szeneanbindung und Ausstieg, auch ob es sich um Frauen oder Männer handelte, wurden sogar erst im Anschluss an die Diskussion der Analyseergebnisse und Forschungshypothesen (durch Fabian Wichmann) zugänglich gemacht. Weitere Informationen zum biografischen Werdegang sowie auch Interna der akuten Fallarbeit wurden den Teilnehmerinnen und der Seminarleiterin nicht offenbart. Neben den angesprochenen methodischen Problemen waren dafür auch rechtliche Gründe ausschlaggebend. Ergebnisse der Analyse auf allen Stufen des Interpretationsprozesses und insbesondere die Hypothesen waren jeweils an der Herkunftsserie zu prüfen (serielle Prüfungen). Über Aussagen zum Einzelfall hinaus erwarteten wir über die Prüfverfahren auch ein vertieftes Verständnis motivischer und stilistischer Ähnlichkeiten des gesamten Untersuchungsbestandes der Aussteiger/innen. Denn aus dem Zusammenhang zwischen innerer und äusserer Bildproduktion war anzunehmen, dass sich allgemeine Charakteristika des Bildbestandes auch als gemeinsame Merkmale des Ausstiegsprozesses interpretieren liessen.

Die Studierenden arbeiteten in kleinen Gruppen von drei bis vier Studierenden mit jeweils einer Fotoserie. Damit und mit dem regelmässigem Abgleich von Zwischenergebnisse im Plenum wurde die für die Analyse von Bildern unabdingbare Multiperspektivität gesichert.

\section{Fotografie als Quelle: Referentialität, Bildhaftigkeit und Medialität}

Die Nutzung von Fotografien als Quelle im Allgemeinen und dieses Fotobestandes im Besonderen erfordert methodologische Vorüberlegungen. Auf das prinzipiell problematische Verhältnis von Intention und Zufall bei der Fotografie wurde bereits hingewiesen, doch muss auch der Doppelcharakter der Fotografie, ihre Referentialität und zugleich Bildhaftigkeit berücksichtigt werden und ausserdem die spezifisch mediale Funktion dieser Fotografien im Rahmen eines vom Fallmanagement initiierten Projektes. Während dem Problem von Intention und Zufall im Projekt «Lebensbilder» wie bereits beschrieben methodisch durch nachfotografische Autorisierung und Kommentierung begegnet wurde, blieb der Umgang mit dem Doppelcharakter der Fotografie schwierig. Denn sie forderte im Rahmen der 
methodisch geleiteten Interpretationen im Seminar immer wieder dazu heraus, das Verhältnis von Indexikalität und übertragener Bedeutung neu zu bestimmen. Denn jedes Foto zeigt eine Aufnahme von etwas Konkretem, aber jedes Foto transportiert auch ein Bild, das konkrete Wirklichkeit nicht einfach wiedergibt bzw. abbildet, sondern das vor dem Objektiv Sichtbare im Prozess der Bildproduktion, wozu wie beschrieben auch nachfolgende Auswahlen gehören, in eine neue bildhafte Realität überträgt. ${ }^{7}$ Das heisst, Fotografien transportieren auch Bilder und evozieren ästhetische Eindrücke über ihre imaginäre Räumlichkeit, die planimetrische Struktur und das enorme metaphorische Potenzial, das sie im Prozess dieser Übertragung gewinnen.

Diesem Spezifikum der fotografischen Quelle soll im Folgenden mit den Begriffen der fotografischen Aufnahme und des fotografischen Bildes Rechnung getragen werden - wohl wissend, dass diese Begrifflichkeiten einer weiteren Qualifizierung bedürfen. Wichtig ist die Differenz, die sie markieren, die sich auch auf die Bestimmung der medial-kommunikativen Funktionen auswirkt. Denn je nachdem in welchem Modus man sich befindet - dem der Aufnahme oder dem des Bildes -, wird man das, was über Fotografien vermittelt wird, anders deuten. Erkennt man in einem Foto zuerst die fotografische Aufnahme, wird man versuchen, etwas wiederzuerkennen, was das Foto zeigt, sich die fotografische Situation vorzustellen, nach der Authentizität der Orte und der Abgebildeten fragen. Im Modus der Bildhaftigkeit wird auch das Wie der Darstellung zum Teil der Bildaussage.

Daher kann uns die Fotografie eines Baumes indexikalisch über seine konkrete Gestalt, den Standort und sein jahreszeitliches Stadium informieren, aber zugleich kann er als Symbol mit einem ausserordentlichen Spektrum an kulturübergreifenden Deutungsmöglichkeiten auf ein Drittes ausserhalb des Bildes verweisen, das eben nicht die Referenz auf das Sichtbare im Moment der Aufnahme ist.

Für die Feststellung des Quellenwertes der Fotografien aus dem Projekt «Lebensbilder» war wichtig, dass man aufgrund der Aufgabenstellung, der Art der Bildgestaltungen, der Auswahl und Autorisierung durch nachträgliche Kommentierung davon ausgehen konnte, dass mit der Bildproduktion ein über die konkrete Abbildung hinausreichender Bildsinn von Seiten der Fotografen/-innen und Bildproduzenten/-innen intendiert war. Aufgabe der Analysen im Seminar war, herauszuarbeiten, was in und mit den Bildern bewusst oder vorbewusst verhandelt und mitgeteilt wurde.

Im Folgenden soll eine ergebnis- und problemorientierte exemplarische Zusammenstellung der im Seminar geleisteten bildwissenschaftlichen Untersuchungen stehen. Der Text dieses Beitrages bildet also nicht die methodisch begründete stufenweise Aufbereitung und Analyse des Bildmaterials im Seminar ab, sondern verdichtet und pointiert Diskussionen und Ergebnisse verschiedener Arbeitsgrup-

7 Das gilt prinzipiell auch für das digitale Foto. 
pen. Ausserdem bietet er aus Gründen der Lesbarkeit eine andere Ordnung der Kontextinformationen, indem biografische Informationen an den Anfang gestellt sind und Bildunterschriften, zum Teil auch Bildkommentare zusammen mit den Abbildungen präsentiert werden. Für die Bewertung der bildanalytischen Arbeit im Seminar und für die Würdigung des Quellenwertes von Fotografien sollte bedacht werden, dass diese Informationen den Studierenden und der Seminarleiterin aus methodischen Gründen während der Bildanalysen nicht zur Verfügung standen.

\section{Ergebnisse seriell-ikonografischer Fotoanalysen im Seminar in Bezug auf Einzelfälle ${ }^{8}$}

Die seriell-ikonografische Fotoanalyse bietet eine Forschungsstrategie, um Fotobestände gezielt auf Forschungsfragen hin zu klassifizieren, Untersuchungsbestände zu qualifizieren und über ein hermeneutisches Verfahren im Rahmen einer auf Fotografie hin modifizierten ikonografisch-ikonologischen Einzelbildinterpretation auszuwerten. Hypothesen, die auf diesem Weg an einzelnen Bildern gewonnen wurden, sind anschliessend seriell am Bestand zu prüfen.

Einige Ergebnisse der im Seminar geleisteten Forschung sollen im Folgenden an zwei Fällen, Tom und Sebastian, exemplarisch vorgestellt werden. ${ }^{9}$ Tom war der jüngste im Projekt «Lebensbilder», 2010 gerade 16 Jahre alt. Er befand sich zu dieser Zeit im akuten Ausstieg, zuvor war er in einer rechtsextremen Gruppe mit drei bis sechs Personen aktiv gewesen und auch kurzzeitig Mitglied der NPD. Sebastian war schon einige Jahre im Ausstieg, er gehörte mit 32 Jahren zu der grösseren Gruppe der Älteren im Projekt.

Toms Foto-Serie (Abb. 1-4) beschäftigte das Seminar wegen ihrer atmosphärischen Dichte und ambivalenter Bildeindrücke, die sie beim ersten Betrachten hervorrief. So bezeichneten einige das Bild (Abb. 1) mit dem Schiff auf offenem Meer als klischeehaft stimmungsvoll, manchen erschien es melancholisch, andere meinten, es wirke still, trostlos und leer.

Im Rahmen des Projektzusammenhangs «Lebensbilder», dem das Foto seine Entstehung verdankt, erscheint es als Teil einer absichtsvollen (visuellen und textförmigen) Äusserung zum Thema Ausstieg, die vom Fotografen selbst durch Auswahl in einen Serienzusammenhang gebracht, betitelt und kommentiert wurde. Welche Aussage darüber formuliert ist, bleibt letztlich hypothetisch, denn Bilder enthalten wie alle ästhetischen Produkte einen nicht einholbaren Bedeutungsüberschuss. Gleichwohl lohnt es, über die im ersten Schritt möglichst kontextfreie Bildanalyse ein breites Spektrum an möglichen (Bild-)Bedeutungen zu erfassen, weil darüber

8 Das methodische Setting der seriell-ikonografischen Fotoanalyse ist ausführlich beschrieben in: Das reflektierte Bild. Die seriell-ikonografische Fotoanalyse in den Erziehungs- und Sozialwissenschaften. (Pilarczyk u. Mietzner 2005).

9 Alle Namen der Teilnehmer/innen am Projekt «Lebensbilder» wurden anonymisiert. 


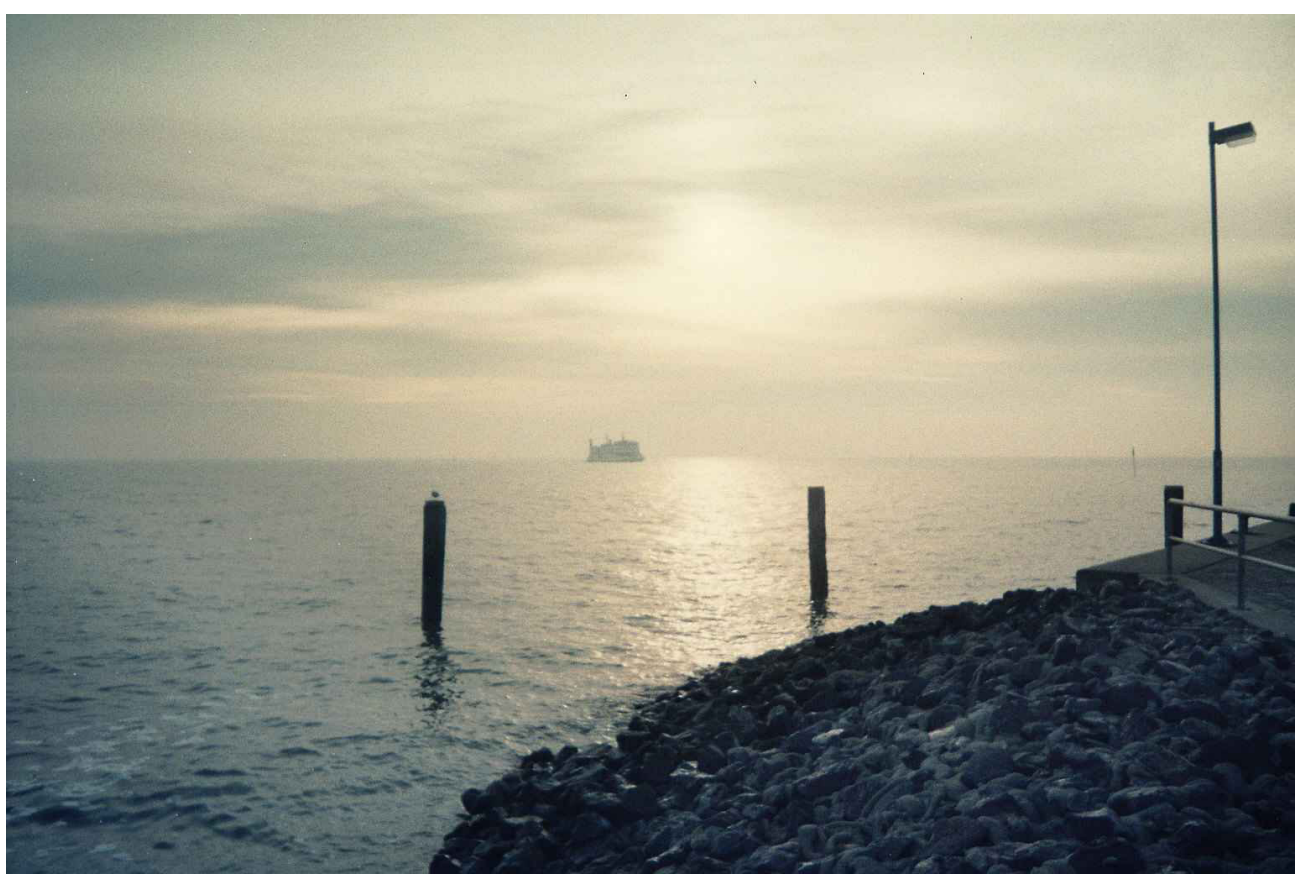

Abb. 1: Tom (anon.), «Hoffnung», analog, Fotoprojekt «Lebensbilder» 2010.

auch jene Bild gewordenen Erfahrungen zugänglich werden, die nicht mit Bedacht adressiert wurden. Der bildliche Kontext (die Serie) und textförmige (Bildunterschriften und Bildkommentare) sind erst daran anschliessend in das analytische Verfahren einzubeziehen. Zu diesem ersten Teil der Analyse (prä-ikonografische und ikonografische Beschreibung) gehören vor allem das detaillierte Beschreiben all dessen, was sichtbar wird und auch das Erfassen der emblematischen und symbolischen Vielfalt von Bildelementen und der ästhetischen Form: u. a. die planimetrische und perspektivisch-räumliche Gestaltung, das Verhältnis von Figur und Grund, von Schärfe und Unschärfe etc.

Als Aufnahme lässt sich das Foto etwa so skizzieren: Der Himmel ist verhangen, Sonnenlicht dringt nur matt hindurch und wird von einer wenig bewegten Meeresoberfläche, die fast Windstille anzeigt, reflektiert. Im Vordergrund sind drei Buhnen (mit dem Umriss eines kleinen Vogels auf der linken) und Steine einer Uferbefestigung erkennbar, rechts ein Metallgeländer, eine Laterne, ein Stück Kaimauer und zwei Metallpoller zum Vertäuen anlegender Schiffe. Der Ausschnitt enthält somit wenige, aber hinreichend spezifische Elemente, sich über die Ränder des Ausschnittes hinaus einen Hafen vorzustellen. Im dunstigen Übergang zwischen Meer und Himmel sind schattenhaft die Umrisse eines Schiffes zu erkennen, das der Form nach vermutlich eine Fähre ist. Die Aufnahme lässt nicht deutlich erkennen, ob sie Kurs aufs offene Meer nimmt oder in Richtung Hafen unterwegs ist. Die 
Funktion der Fähre, ihr Hin- und Her ist damit an jenem Punkt erfasst, wo sich eine Richtung nicht bestimmen lässt.

Über die Bildlichkeit der Fotografie, also Foto als Bild, vermittelt sich durch die Horizontlinie fast exakt in der Mitte und die Anordnung wichtiger Feldlinien im rechten Winkel etwas Statisches, es gibt keine bildbestimmenden Fluchtlinien oder Fluchtpunkte. Im Kontrast zur Weite der Himmels und Seefläche wirken die dunklen Silhouetten der Buhnen samt Vögelchen vereinzelt, das Ensemble am rechten Bildrand verlassen. Die Fläche der Steine im rechten Vordergrund tritt massiv und dunkel hervor. Das Motiv Schiff befindet sich im geometrischen Zentrum des Bildes, das aber, weil es dort nur schemenhaft erkennbar ist, andeutungsvoll auf eine fragile und widersprüchliche Existenz verweist: ist es gerade dabei hervorzutreten oder verschwindet es? Als Bildmotiv trägt Schiff/Fähre hier potenziell eine Vielfalt symbolischer Deutungen mit ein, noch viel mehr Meer, Wolken, Sonne/Licht und Steine, die grosse Bildflächen dominieren. Über die visuellen Formulierungen des ruhigen, aber undurchdringlichen Meeres, eines unklaren, wolkenverhangenen Himmels und des zerstreuten und gespiegelten Sonnenlichts, wird eine starke atmosphärische, vieldeutige Grundstimmung erzeugt, die Rückschlüsse auf Sichtweise oder gar Intentionen des Fotografen spekulativ erscheinen lassen - nicht zuletzt deshalb, weil sie Betrachter/innen gerade dadurch herausfordert, eigene Stimmungen und Erfahrungen in die Rezeption des Bildes mit einzubringen.

Daher war wichtig, Toms visuelle Äusserung daran anschliessend im Zusammenhang der Serie zu betrachten und damit in den grösseren bildlichen Kontext der gesamten visuellen Äusserung einzuordnen. In diesem Zusammenhang zeigten sich Übereinstimmungen, alle Bilder wirkten statisch, die Farbgebung war diffus und - alle Bilder verbindet ein eigentümlicher Stil. Schnell identifizierbare fotografische Motive (Schiff, Wege, Baum) dominieren die Bildzentren, aber erscheinen in der Art der Darstellung widersprüchlich: Wege führen nirgendwohin, Ausblicke oder Ziele sind im Bild nicht formuliert und der Baum ist eigentlich ein Baumstrauch. Grössere, bildbestimmende Bildflächen entziehen sich detaillierter Bildbeschreibung, lassen sich nur begrifflich zusammenfassend indexikalisch erfassen: Meer und Himmel, Schneedecke und Schneereste, Gestrüpp, Verästelungen, aufgeworfene Erde und alte Pflanzenreste. Fluchtpunkte, wo es sie gibt (Abb. 2 und 3), bleiben unbestimmt, die Bilder entzogen sich eindeutiger Interpretationen. Die Bildräume und atmosphärischen Stimmungen konnten, jedes Bild für sich genommen (so wie Abb. 1), stimmungsvoll wirken, im Zusammenhang der Serie vermitteln die motivischen und stilistischen Bildarrangements jedoch auch den Eindruck von Leblosigkeit und Stille. Toms Bildunterschriften «Hoffnung», "Brücke», «Angst», «Einsam» hingegen stiften eine dramatische Spannung, die die Bilder ohne sie nicht haben. 


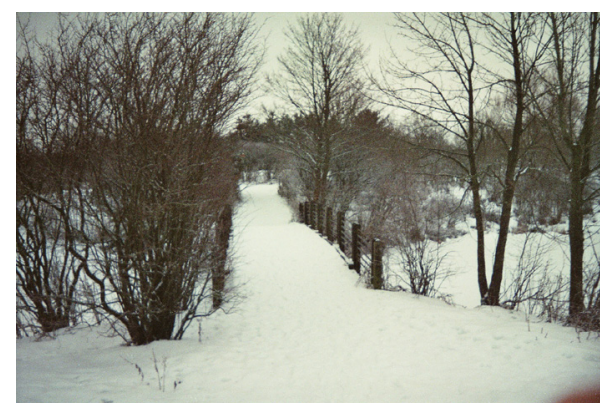

Abb. 2: Tom (anon.), «Brücke», analog, Fotoprojekt «Lebensbilder» 2010.

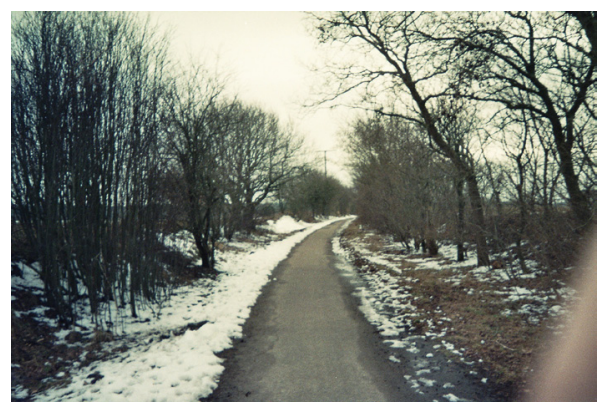

Abb. 3: Tom (anon.), «Angst», analog, Fotoprojekt «Lebensbilder», 2010.

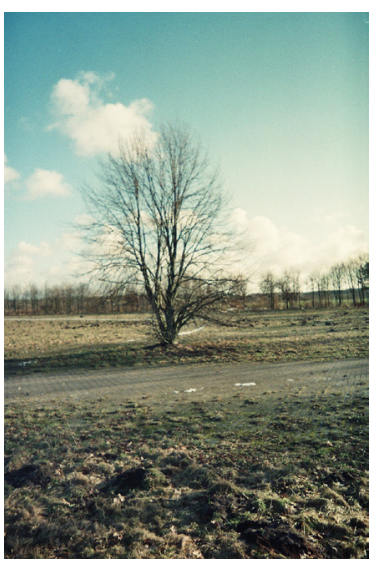

Abb. 4: Tom (anon.), «Einsam», analog, Fotoprojekt «Lebensbilder», 2010.

Um zu verstehen, was in den atmosphärisch eigentümlichen, mehrdeutigen und auch in Bezug auf die Unterschriften widerspruchsvollen Bildern verhandelt wurde bzw. um dies auf die Lebenssituation des Jungen hin deuten zu können, wurde im Gang der weiteren Untersuchung entschieden, auch Toms Textkommentierung in die Analyse mit einzubeziehen.

Dabei konnte es nicht einfach darum gehen, den Kommentar auf das Bild zu beziehen, erprobt wurde eine Kontrastierung von Text- und Bildanalyse. Faktisch stellt dies eine methodische Erweiterung der seriell-ikonografischen Fotoanalyse dar. Denn zwar war dort bislang im Rahmen von Geltungsprüfungen auch angelegt, Textdokumente mit einzubeziehen, doch neu war, die Textkommentare ebenfalls quellenkritisch, textanalytisch zu untersuchen und erst dann mit den Ergebnissen der Bildanalysen zu kontrastieren.

Dieses methodenerweiternde Vorgehen erwies sich deshalb als sinnvoll, weil sich in der Opposition von Bild und Text nun auch Toms Motivwahlen und sein ästhetischer Stil als Strategie seines ganz persönlichen Umgangs mit der Situation (im Ausstieg) offenbarten.

So kommentiert er das bereits vorgestellte Bild mit dem Schiff (Abb. 1):

Hoffnung auf eine schönere, bessere und lange Zukunft ohne Probleme und ohne Hass. Hoffentlich kommt dieser Tag bald, an dem alles besser wird. (EXIT-Deutschland 2011, 66)

Die Hoffnung auf Zukunft bleibt in dieser Formulierung begrifflich abstrakt, Toms Kommentar lässt nicht einmal erkennen, ob es die eigene Hoffnung war, von der die Rede ist. 
Analog verwendet er im Bild für Hoffnung (mehr noch für Sehnsucht) klischeehaft genutzte, also recht allgemeine visuelle Formulierungen von Meer, Schiff, Horizont. Der Mangel an Konkretion zeigt sich bei näherer, nämlich textanalytischer Betrachtung auch in Toms Bildkommentar. Der Jugendliche formuliert mit einer gewissen Eloquenz doch ohne Subjekt und Prädikat, offenbar hat er eine unbestimmte Vorstellung wie Zukunft (für ihn) aussehen soll: «eine schönere, bessere, lange». Auch formuliert er nicht, was er tun will oder wird, vielmehr bezieht sich der Terminus «Hoffnung» vage auf einen Zeitpunkt, der (hoffentlich) auf ihn (bald) zukommt. Text und Bild stimmen im Modus der unklaren Aussage, der Indifferenz und eines unbestimmten Wartens überein: Der Tag kommt (vielleicht) sowie das Schiff sich (vielleicht) deutlicher zeigt, wenn sich der Himmel klärt. Dass es auch genau andersherum sein kann, dass das Schiff verschwindet, wird zumindest im Bild wie bereits beschrieben, offen gelassen.

Auch in anderen Kommentaren vermeidet Tom eine persönliche Stellungnahme. Zu dem Bild «Brücke» (Abb. 2) schreibt er:

Dankbarkeit dafür, dass es für Personen, die auf der falschen Seite stehen, immer noch Hilfe geben kann, damit sie auf einen neutralen, harmlosen Weg kommen können. Aber dafür müsste man erst die Brücke überqueren, was bestimmt für viele Überwindung kostet, um dem Alten den Rücken zuzukehren und um für das Neue offen zu sein. (EXIT-Deutschland 2011, 66)

Wieder ist nicht klar, wer eigentlich spricht, zugleich werden vordergründig Themen verhandelt, von denen man weiss, dass sich Aussteiger damit beschäftigen: auf den Weg kommen, die richtige Seite wählen, Hilfe bekommen, sich überwinden, Offenheit für Neues usw. Formulierungen wie die eines «neutralen, harmlosen Weges» sind aber für persönliche Zielbestimmungen ebenso wenig tauglich wie das unbestimmte «man» «kann» und «müsste» «viele».

Die Art und Weise, wie Tom Themen verbal und bildlich formuliert, offenbart nicht reflektierte Auseinandersetzung, sondern erscheint als atmosphärisches Rauschen, in dem fällige oder vielleicht auch erwartete Themen angedeutet sind, aber nicht ausgearbeitet werden.

Die Hypothese, die sich in Bezug auf die Lebenssituation des Jungen nach dem Zusammenführen von Bild- und Textanalyse aufstellen lässt, war: Themen und Motive, fotografische ebenso wie die des Handelns, scheinen eine strategische Funktion zu haben, um einen aktuellen Zustand, man könnte ihn depressiv nennen, zu verschleiern.

Dieser Befund machte die Studierenden betroffen, weil sich dieses Untersuchungsergebnis weder mit ihren eigenen Vorstellungen vom Aussteigen noch mit objektiven Kriterien für einen erfolgreichen Ausstieg vereinbaren liess. 
Das zweite Foto, das hier eingehender besprochen werden soll, stammt von dem zu diesem Zeitpunkt über 16 Jahre älteren Sebastian. Er wurde in Ostdeutschland geboren und war mehr als zehn Jahre in der gewaltbereiten-militanten Neonaziszene aktiv, u.a. als Mitglied in mehreren Kameradschaften. Er organisierte die Haftbetreuung von Rechtsextremisten, nahm an Aufmärschen teil, dokumentierte und archivierte sämtliche Aktivitäten der lokalen Szene und publizierte in rechtsextremen Fanzines. ${ }^{10}$ Sebastian ist wegen Körperverletzung vorbestraft.

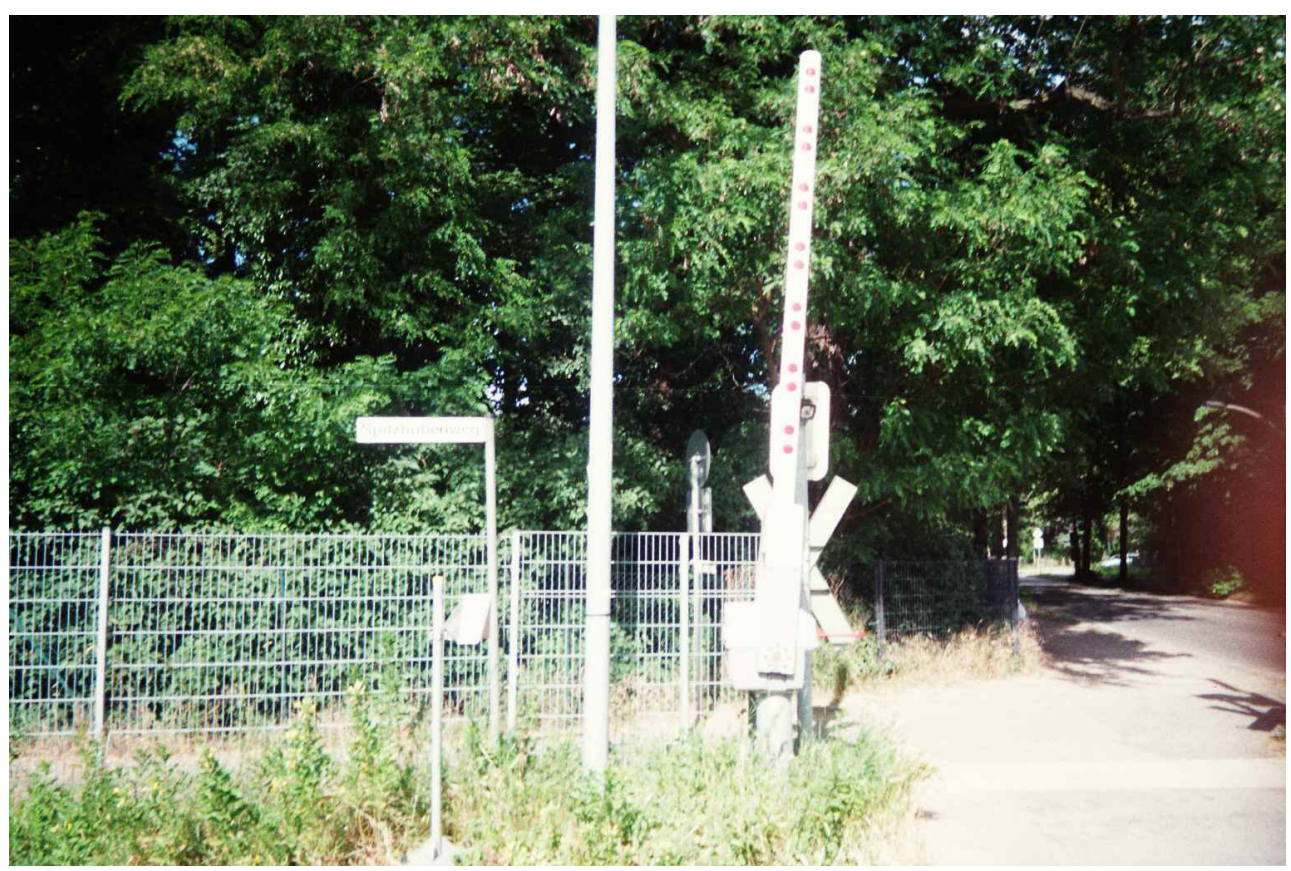

Abb. 5: Sebastian (anon.), «Umsetzungsagentur für gelebte Demokratie (Fug) aktuell», analog, Fotoprojekt «Lebensbilder», 2010.

Was an diesem Foto-Bild (Abb. 5) zuerst auffiel, war der Mast, der das Bild in zwei fast gleich grosse Hälften aufteilt und damit zwei Bilder generiert, in denen der Fotograf visuell zwei unterschiedliche Weg-Optionen formuliert. Die linke Bildhälfte führt den "Spitzbubenweg» am Zaun entlang, eine räumliche Perspektive ist in diesem Teil nicht gegeben. ${ }^{11}$

Die rechte Seite gibt den Blick im Bild frei durch die geöffnete Schranke auf eine Strasse unter Bäumen. Wohin sie führt, ist auch hier ungewiss. Diese Hälfte ist

10 Fanzines sind Magazine, die von Fans für Fans gemacht werden. Inhaltlich orientieren sich die Magazine an der jeweiligen Subkultur oder einzelnen Bands. In der rechtsextremen Szene erfreuen sich solche Publikationen grosser Beliebtheit, da über diesen Weg szeneintern kommuniziert und Informiert werden kann.

11 Dass sich der Fotograf der übertragenen Bedeutung von «Spitzbubenweg» für sein Bild durchaus bewusst war, belegt ein zweites Foto aus seinem Bestand, das dieses Strassenschild als zentrales Motiv hat. 
durch ein Stück des Zaunes mit der linken verbunden, der Fotograf steht vor der Schranke. Geht er weiter, ist eine Wegentscheidung zu treffen, zwar ist die Schranke geöffnet, aber Vorsicht ist geboten (sie kann sich auch schliessen), das Andreaskreuz verstärkt die Warnung.

In seinen ausführlichen Bildkommentaren warnt Sebastian u. a. vor der «revolutionären Sackgasse», in die er selbst geraten war. Aber wenn man es schaffte, das «innere Navigationssystem» auszubauen, «kann mit einiger Verspätung dann die innere Bestätigung ‘Sie haben ihr Ziel erreicht!) eintreffen, doch besser später, als nie und nimmer!» (unv. Projekt «Lebensbilder» 2010). Bilder und Texte lassen einen nachdenklichen Menschen erkennen, der mit Entschiedenheit und metaphorischer Kraft zu formulieren vermag (siehe auch Abb. 12 und 17), was die Studierenden als Fähigkeit zum reflektierten Umgang mit der eigenen Biografie und der Situation des Ausstiegs deuteten. Ein weiteres Ergebnis der Analyse war, dass er sich bildhaft und auch in den Kommentaren hauptsächlich mit dem Thema Beschränkung, Einengung, Umzäunung und den Wegen (hinein und hinaus) auseinandersetzt.

Wir, die sich selbst eingezäunt haben, wollen denen ausserhalb erklären, dass sie sich von Unterdrückung und Unfreiheit befreien müssen, führen wir vielleicht, ohne dass es uns bewusst wird, Selbstgespräche? Sind wir vielleicht die eigentlichen Empfänger unserer Botschaft? (unv. Projekt «Lebensbilder» 2010)

\section{Ergebnisse seriell-ikonografischer Fotoanalyse im Seminar in Bezug auf den Ausstieg als Prozess}

Die Themen, die Tom und Sebastian beschäftigen, Wegsuche und Beschränkung waren offensichtlich, das zeigten die seriellen Untersuchungen, allen Aussteigern/innen besonders wichtig. Motivisch zeigt sich das wie bei Tom und Sebastian u. a. in einer Häufung der Wegemotivik.

Das ist vielleicht nicht besonders überraschend bei einer Personengruppe, die auf der Suche nach Orientierung ist. Doch ist interessant, wie die Wege jeweils visuell umgesetzt sind, wo sie hinführen und welche Stimmung die darüber gestalteten Bildräume vermitteln.

In ihren Bildern erscheint das Motiv Weg vielgestaltig: als kaum befestigter Waldweg (Abb. 6); als asphaltierte Strasse geradlinig mit einer gewissen Dynamik (Abb. 7); als Bürgersteig in urbanem Milieu (Abb. 8); als bewegter Wasserweg (Abb. 9); als Schienenweg durch die (grosse) Stadt, hier entweder perspektivisch mit dem Blick des Betrachters in den Bildraum hinein (Abb. 10) oder fast parallel als dyna- 


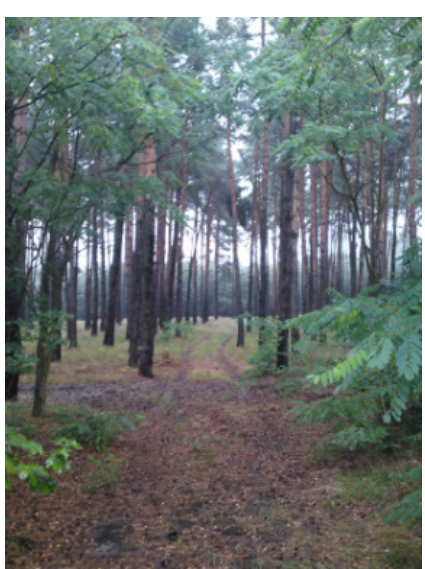

Abb. 6: Andreas (anon.), «Waldweg», analog, Fotoprojekt «Lebensbilder», 2010.

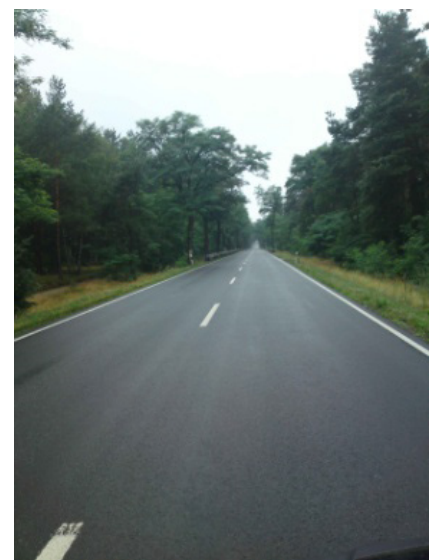

Abb. 7: Andreas (anon.), "Strasse», analog, Fotoprojekt «Lebensbilder», 2010.

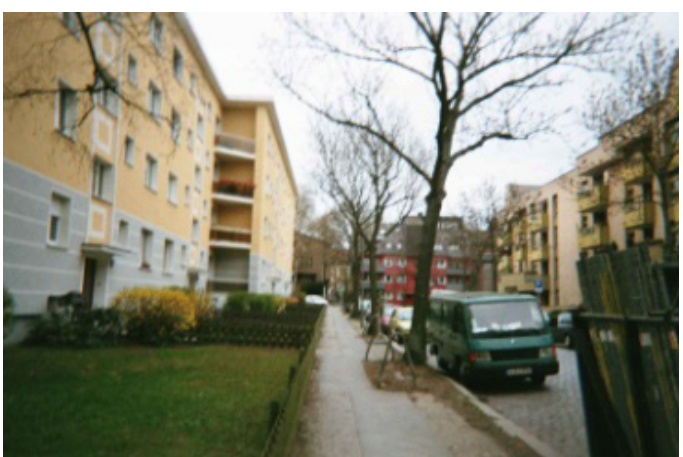

Abb. 8: Steffen (anon.), «O. T.», analog, Fotoprojekt «Lebensbilder», 2010.

misch das Bild schneidende Linien (Abb.11). Gemeinsam ist diesen Bildern und den darin formulierten Wegen, dass diese nicht klar auf ein Ziel hin entworfen sind, sondern zumeist als Möglichkeit, eine bestimmte Richtung einzuschlagen.

Auch auf der Textebene erschliesst sich die zentrale Bedeutung des Weges für die Aussteiger/innen, z. B. in Marcels Kommentar zu Abb. 10: «der neue Weg im Licht» (EXIT-Deutschland 2011, 8), Karen zu Abb. 9: «(a)uch erst mal einen Weg finden» (Karen, ebd., 19). Sebastian schrieb zu einem hier nicht abgedruckten Foto: «Freiheit ist im Allgemeinen keine abgesteckte Wegstrecke, sondern eine sehr breite und ausgreifende 〈Allee〉, mit sinnbildlich unendlich vielen Büschen, Sträuchern,

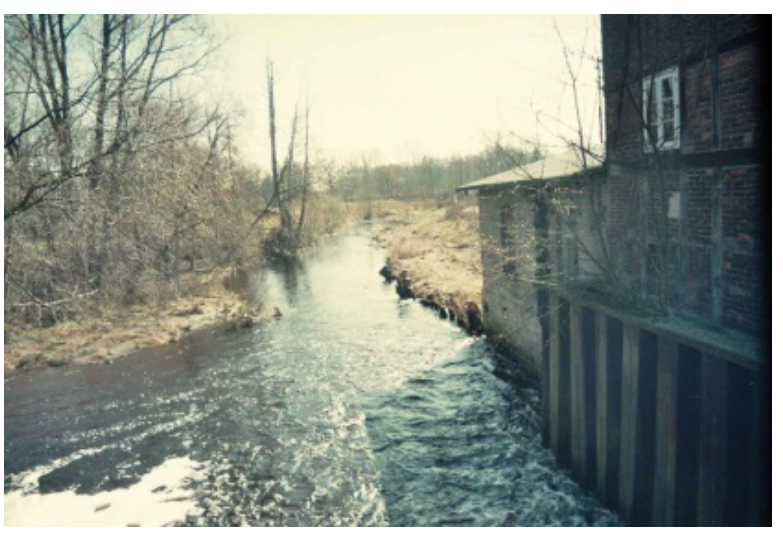

Abb. 9: Karen (anon.), «Flusslauf», analog, Fotoprojekt «Lebensbilder», 2010.

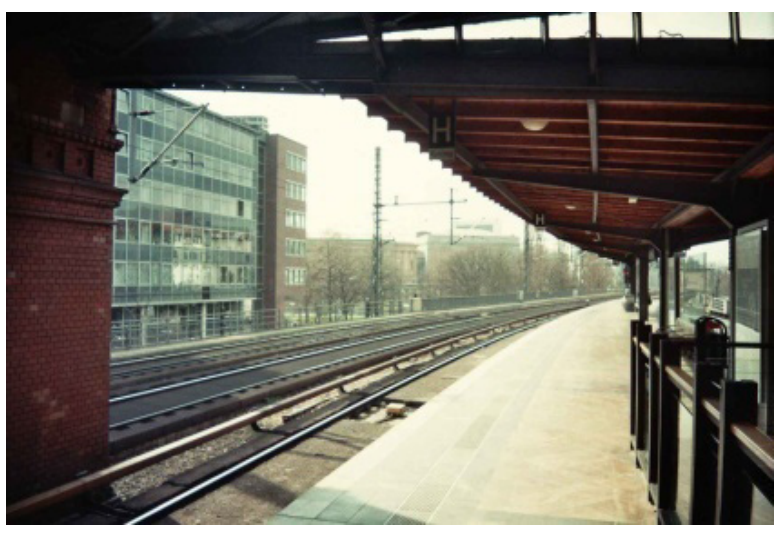

Abb. 10: Marcel (anon.), "Neue Wege», analog, Fotoprojekt «Lebensbilder», 2010 


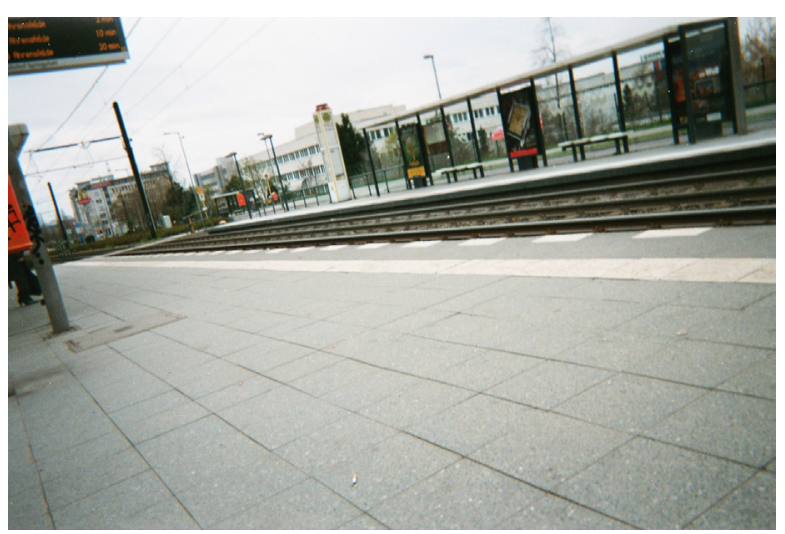

Abb. 11: Steffen (anon.), «Abfahrt: Eingefahren, Abgefahren, Ausgestiegen - Endstation? Offen geblieben», analog, Fotoprojekt «Lebensbilder», 2010.

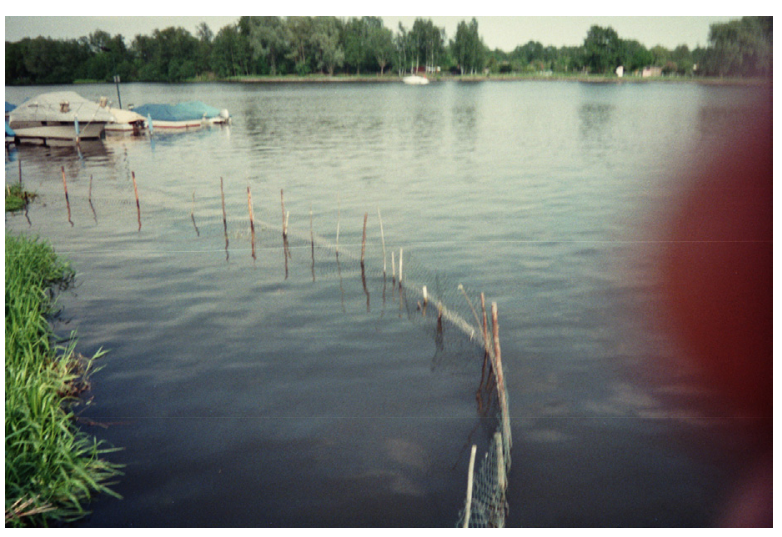

Abb. 12: Sebastian (anon.), «Flussabschnitt eingezäunt», analog, Fotoprojekt «Lebensbilder», 2010.

Bäumen und anderen wie auch immer gearteten «Gewächsen»» (unv. Projekt «Lebensbilder» 2010).

Der Weg ist natürlich auch dort präsent, wo explizit oder implizit (Aus-) bzw. Weglosigkeit verhandelt wird (Abb. 5, 6, 17, 18), dann ist das Wegemotiv auch Teil des Themas Beschränkung. Motivisch ist Beschränkung einerseits als Zäune, Mauern, Begrenzungen usw. präsent. (Abb. 1, 2, 5, 10-15, 17, 18)

Darüber hinaus wird Beschränkung über Lenkung und Blickhinderungen unmittelbar ästhetisch nacherfahrbar, z. B. wenn der Blick an bildbestimmenden Flächen und Strukturen förmlich abprallt (Abb. 14) oder eingefangen und (Abb. 15) gelenkt wird.

Wie die Wegsuche wird Beschränkung auch in den Bildkommentaren verhandelt, z. B. wie bei Sebastian s. o., auch Steffen zu Abb. 15: "Gitter, Stäbe und Mauern

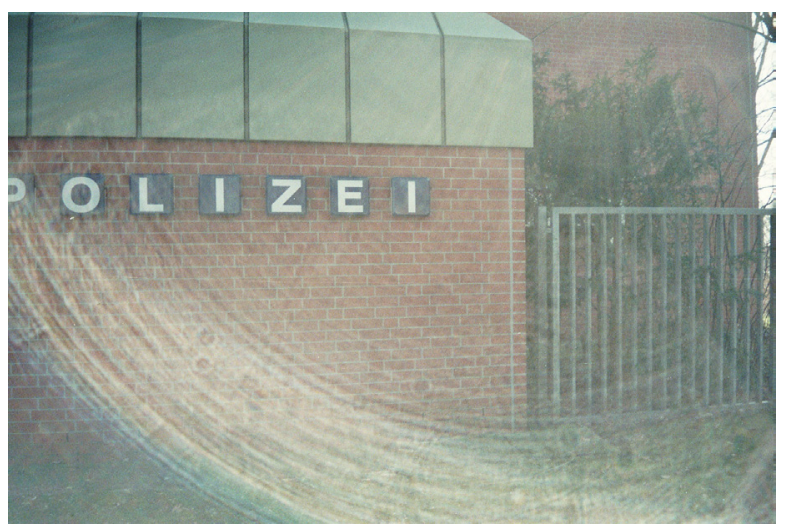

Abb. 13: Karen (anon.): «Polizei», analog, Fotoprojekt «Lebensbilder», 2010.

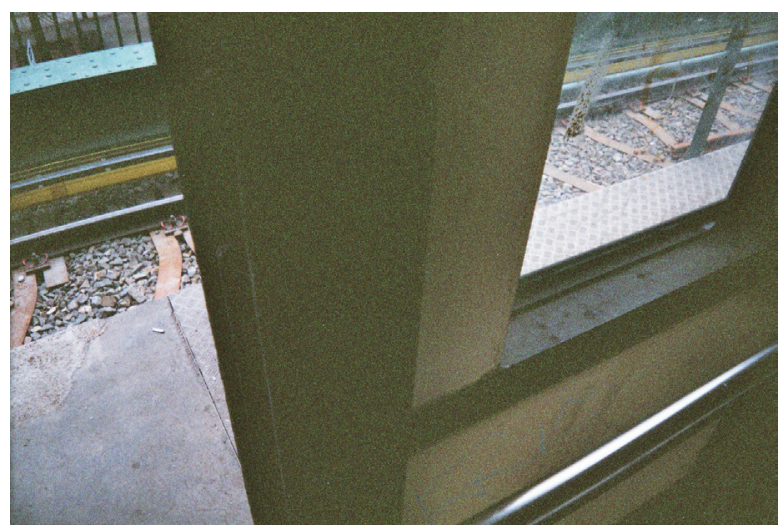

Abb. 14: Marcel (anon.), o. T., analog, Fotoprojekt «Lebensbilder», 2010. 


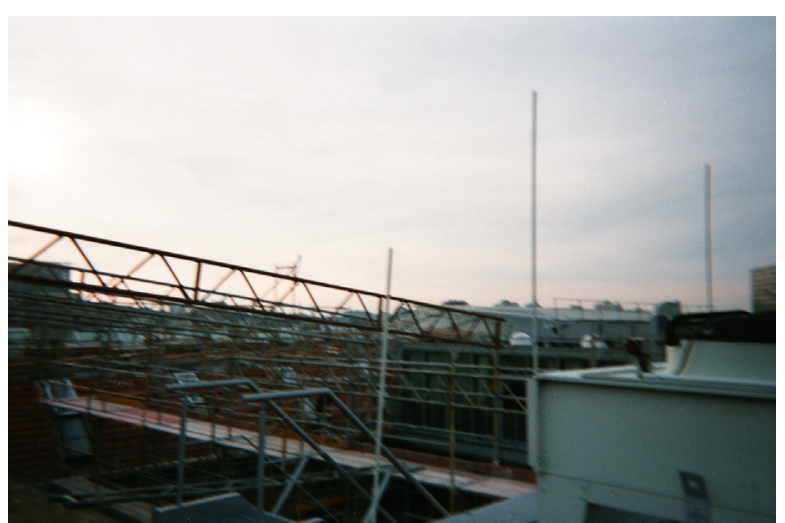

Abb. 15: Steffen (anon.), «Knast», analog, Fotoprojekt «Lebensbilder», 2010.

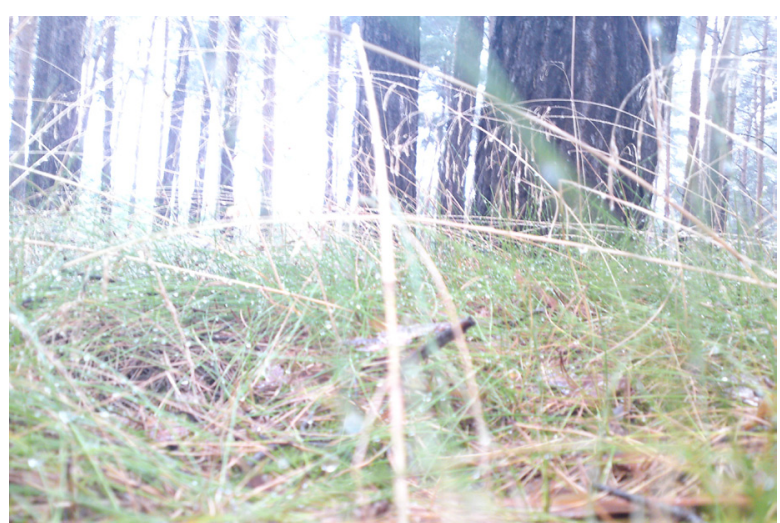

Abb. 16: Andreas (anon.), «Halm», analog, Fotoprojekt «Lebensbilder», 2010.

verschränkten mir die Sicht. Auch jetzt habe ich sie täglich vor mir, aber die Perspektive bestimme nun ich.» (EXIT-Deutschland 2011, 41), oder Andreas zu einem hier nicht abgedruckten Foto: «Der Ausbruch ist schwer, ich sehe die Abgrenzung, ich sehe die Spitzen auf dem Zaun» (ebd., 56).

Zwei Fotografien, eine von Sebastian (Abb. 17), die andere von Karen (Abb. 18), führen die unterschiedlichen motivischen und stilistischen Formulierungen zum Thema Beschränkung auf spezifische Weise und ästhetisch eindrucksvoll zusammen.

Sehen wir diese Fotografien als Aufnahmen, um die anfänglich beschriebene fotospezifische Unterscheidung von Aufnahme und Bild noch einmal aufzunehmen, können wir indexikalisch jeweils einen Maschendrahtzaun identifizieren, durch den hindurch die Fotografen, Sebastian und Karen, die dahinter liegenden Motive aufgenommen haben. Betrachten wir die Fotografien als Bilder, fungieren die Strukturen der Zäune als bildbestimmende Muster, die Betrachter/innen zwingt, das Dahinterliegende wie durch einen Filter wahrzunehmen. Die Grundform dieses aus symmetrischen Wiederholungen resultierenden Musters ist die Raute, bei der sich ohne Mühe Assoziationen von Odalrunen einstellten, die sich in diesem Muster fortsetzen. In Sebastians Bild verstärkt das wiederkehrende Muster das Arrangement von Transformatoren, Leitungen und Gitter, deren energetische Spannung sich den Betrachtern/-innen vermittelt. Zugleich formieren sich diese Elemente im Schatten des Sonnenlichts auf der Bildebene wie von selbst auf der Bildebene zu Hakenkreuzen. Der Maschendrahtzaun trennt den Fotografen von dem, was sich hinter dem Zaun befindet, im Bild schafft er Distanz zwischen der eigenen Position und das Muster zeigt an, was der Ort symbolisiert, seine (Nazi-)Vergangenheit. Auf diese Zeit und seine mentale Verfassung damals bezieht sich auch Sebastians ironischer Bildtitel. 


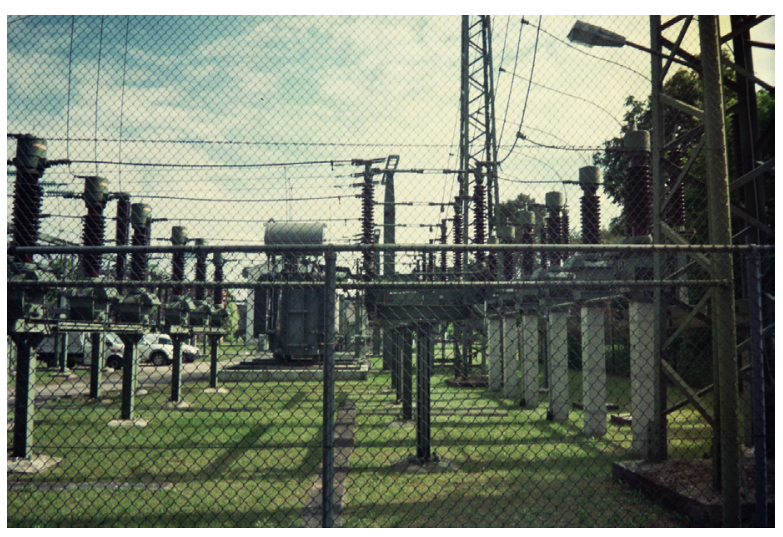

Abb. 17: Sebastian (anon.), «Immer unter Hochspannung, immer unter Volldampf», analog, Fotoprojekt «Lebensbilder», 2010.

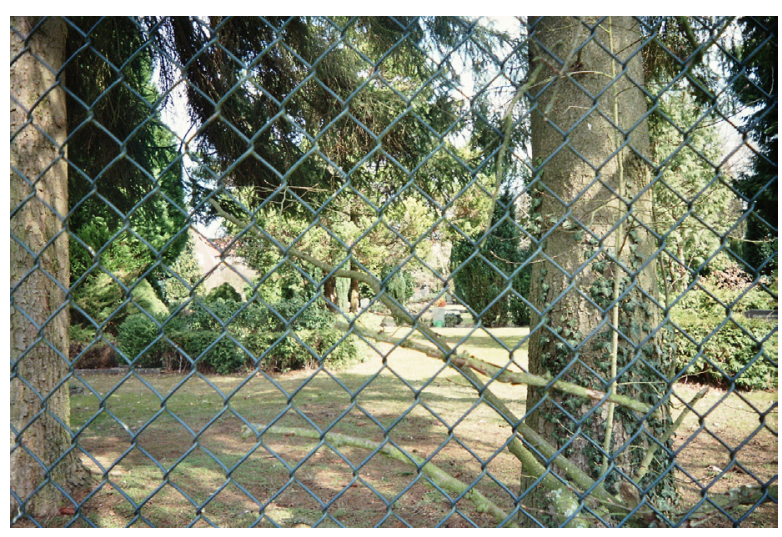

Abb. 18: Karen (anon.), «Der innere Tod», analog, Fotoprojekt «Lebensbilder», 2010.

Karens Bild hingegen ist weniger spannungsreich, fast elegisch. Was hinter dem Zaun liegt, erscheint auch nicht als etwas Abgeschlossenes, mit dem sie nun wie Sebastian abrechnen könnte. Dazu ist dieser Ort zu schön, er erscheint in ihrem Bild eher als ein Ort, der Ruhe verheisst. Doch ist er, so sonnig und grün er auch scheint, dennoch erkennbar ein Friedhof. Der Zaun trennt sie von diesem Ort und ein Wahrnehmen und Beschreiben des Bildes ist nur durch diesen Filter möglich.

Die von den Studenten/-innen herausgearbeitete Funktion dieses Musters als Wahrnehmungsfilter war für sie auch eine Erklärung für die Orientierungslosigkeit, die sich über Karens andere Bilder und auch in Kommentaren ausdrückt. Das führte sie zu der Hypothese, dass diese Aussteigerin vermutlich mit dem Problem kämpft, ihre Situation deutlich zu erkennen, angemessen einzuschätzen und Schlüsse für ihr Handeln zu ziehen. Den hier visuell formulierten «Ort der Ruhe» deuteten sie zugleich als Ausdruck grosser Erschöpfung.

Bezogen auf den Ausstieg als Lebenssituation und Prozess der ganzen Gruppe zeigten die motivischen und stilistischen Ähnlichkeiten im Bestand des Fotoprojektes «Lebensbilder» nach der Analyse für die Studierenden im Sommersemester 2011 eher Unerwartetes und sie machten sie nachdenklich.

Wege ohne Ziel, Zäune, Unschärfe und gitterartigen Strukturen, die das Erkennen erschweren und Blicke verstellen, standen im Widerspruch zu dem, was Ausstieg bei Nichtbetroffenen häufig assoziiert: Befreiung, Neuanfang und den Gewinn neuer Perspektiven. Zäune und Blickbegrenzungen können hingegen als Auseinandersetzung mit den aktuellen und überwundenen Beschränkungen der Situation interpretiert werden, die auffällig starke Strukturierung fast aller Bilder auch als ein Bedürfnis nach Ordnung und Halt, das die Situation des Ausstiegs offenbar hervorbringt. Unschärfe und Ziellosigkeit resultieren - so die Hypothesen - aus der Unfähigkeit, klare Formulierungen auf Zukunft hin zu finden. 
Die thematische Konzentration auf Weg und die motivischen und stilistischen Umsetzungen des Themas wurden im Anschluss an die Analysen als Wegsuche verstanden, wohlbemerkt nicht als Zielbewusstsein, sondern erst einmal als ein auf dem Weg sein, nicht mehr und nicht weniger. Und manche dieser Bilder und Kommentare drücken dann auch eher eine Hoffnung auf Zukunft aus als die Gewissheit, eine zu haben. Für die meisten der Aussteiger/innen scheint Zukunft nur erst ein Wort zu sein, das in den Kommentaren zwar als Wort auftaucht, zu dem aber noch die bildlich konkreten Vorstellungen, d. h. auch der Begriff fehlt.

Die Bilder bringen damit Unwägbarkeiten, Unbewusstes, manchmal nur energetisch zu Fühlendes zum Ausdruck. Das aber, so war die zusammenfassende Einschätzung des Seminars, kann uns über die Analyse und den ästhetischen Nachvollzug helfen, den Ausstiegsprozess als komplex und widersprüchlich zu begreifen, der nicht mit Befreiung und endlich im richtigen Leben angekommen sein beginnt, sondern mit enttäuschten Hoffnungen, Scham und unerfüllter Sehnsucht nach Zugehörigkeit. Und er scheint sich als permanente, zunächst einsame und individuelle Wegsuche zu gestalten, die Unterstützung braucht und die dennoch nicht in jedem Fall gelingt.

Gerade in Bezug auf den Fall Tom machte Fabian Wichmann in der Abschlussdiskussion auch deutlich, dass die Grenzen der sozialpädagogischen Intervention dort erreicht sind, wo Therapiebedarf angezeigt ist. Dass der Ausstieg auch scheitern kann, müssen wir akzeptieren. Hierbei helfend und beratend zur Seite stehen zu können, erfordert Verständnis und Sensibilisierung für die allgemeinen Charakteristika des Prozesses und die Individualität des Ausstiegs. Das schafft Analyse nicht allein, sondern hier bedarf es lebendiger Nacherfahrung, und diese ermöglicht, so waren wir nach den Analysen überzeugt, vielleicht am ehesten die künstlerische Produktion. Aus dieser Erkenntnis entstand die Idee eines anschliessenden Seminars an der TU-Braunschweig im folgenden Jahr, mit dem eine kreative, künstlerisch-ästhetische Auseinandersetzung mit dem Bild- und Textmaterial der Aussteiger/innen initiiert und über eine Ausstellung vermittelt werden sollte.

\section{3 «Bilder und Texte von Aussteigern aus dem Rechtsextremismus» - eine Ausstellung (Sommer-Semester 2012 auf dem Campus Nord der TU- Braunschweig; Ulrike Pilarczyk)}

Intention eines im Sommersemester 2012 an diese Arbeiten anschliessenden Seminar-Workshops war daher, über den Nachvollzug der bildanalytischen Ergebnisse des Vorjahres und durch die Initiierung einer ästhetisch-künstlerischen Auseinandersetzung mit den bildlichen und sprachlichen Äusserungen der Aussteiger/innen diese in eine verdichtete ästhetische Form zu bringen, um die Analyseergebnisse anderen Menschen anschaulich vermitteln zu können. Der Ausstiegsprozess sollte über die Konzeption und Gestaltung einer Ausstellung einer breiteren Öffent- 
lichkeit zugänglich gemacht werden. Unser Anliegen hatte unterdessen durch das Bekanntwerden der NSU-Morde im Herbst 2011 eine zusätzliche, traurige Brisanz erhalten.

Ging es im Foto-Projekt «Lebensbilder» 2010 unmittelbar um biografische Reflexion und sozialpädagogische Intervention, im Seminar 2011 um den Erwerb bildwissenschaftlich methodischer und fachlicher Kompetenzen durch die Studierenden, sollte in dieser dritten Phase 2012 eine (museums-)pädagogische Auseinandersetzung und Vermittlung stattfinden. Auch diese Phase wurde unter Leitung von Ulrike Pilarczyk in Kooperation mit EXIT-Deutschland und zusammen mit Fabian Wichmann als Seminar-Workshop gestaltet.

Die ästhetischen Lösungen, die die Seminarteilnehmerinnen nach einer Phase der Informationsverarbeitung (der Analysen vom Vorjahr), Diskussion und Orientierung fanden, waren Kontrastierungen von Bild und Text im Rahmen eigener bildhafter Gestaltungen. Dafür standen ihnen Bild-Schnitt, Textauswahl und Anordnung von Bild und Text in und um den Rahmen der Originalbilder herum zur Verfügung, weitere Veränderungen der Originalbilder etwa durch Übermalungen, waren nicht gestattet. $^{12}$

Mit welchem Ergebnis diese Auseinandersetzung stattfand, soll im Folgenden wegen des hier zur Verfügung stehenden Raumes skizzenhaft an Bearbeitungen der zwei bereits vorgestellten Fotos und Texten von Tom und Sebastian vorgestellt werden.

Wie bereits beschrieben, hatten die Studierenden des Vorjahres über die Analyse der Foto-Serie und der Kommentare von Tom herausgearbeitet, dass das eigentliche Thema seines Bildes (Abb. 1) nicht wie von ihm betitelt «Hoffnung» ist, sondern dass Bild- und Textausdruck gleichermassen massive Probleme offenbaren, die ihn hindern, den Ausstieg erfolgreich voranzutreiben. Die Studierenden im Sommersemester 2012 fanden, um dies zu vermitteln, eine Lösung, die nach ihrer Meinung die mentale Disposition des Jugendlichen auf den Punkt und ästhetisch eindringlicher nacherfahrbar macht als seine Fotografien und Kommentare, die seine Situation auf den ersten Blick ja eher verdecken.

Für die Ausstellung fügten die Studierenden beide Ausdrucksebenen zu einer neuen bildhaften Darstellung zusammen (Abb. 19). Dafür beschnitten sie sowohl Toms Bild als auch seinen Kommentar dazu (Abb. 1) «Hoffnung auf eine schönere, bessere und lange Zukunft ohne Probleme und ohne Hass». Der "Hoffnung»

12 Zeitlich und finanziell überschritt das Projekt die Möglichkeiten eines Seminar-Workshops. Für die technische Umsetzung dieses Projektes konnten Gelder des Innovationsfonds Lehre der Fakultät 6 der Universität eingeworben werden. Im Seminar erarbeiteten die Studierenden Entwürfe in PowerPoint im Bildverhältnis der geplanten Ausstellungsgrösse, die den Rahmengrössen der Fenster der Glasfront im Hörsaalgebäude des Campus Nord entsprechen sollten. Eine Layouterin brachte die Entwürfe auf diese Grösse von ca. 180x80, anschliessend wurden die Bilder auf Forex-Platten gedruckt. Die Seminarteilnehmer/innen brachten die Platten an die Wände des Hörsaal-Gebäudes an und klebten sie in die Fensterrahmen ein. An jeder Tafel hing ein Kärtchen mit den biografischen Daten der Aussteiger/innen. 


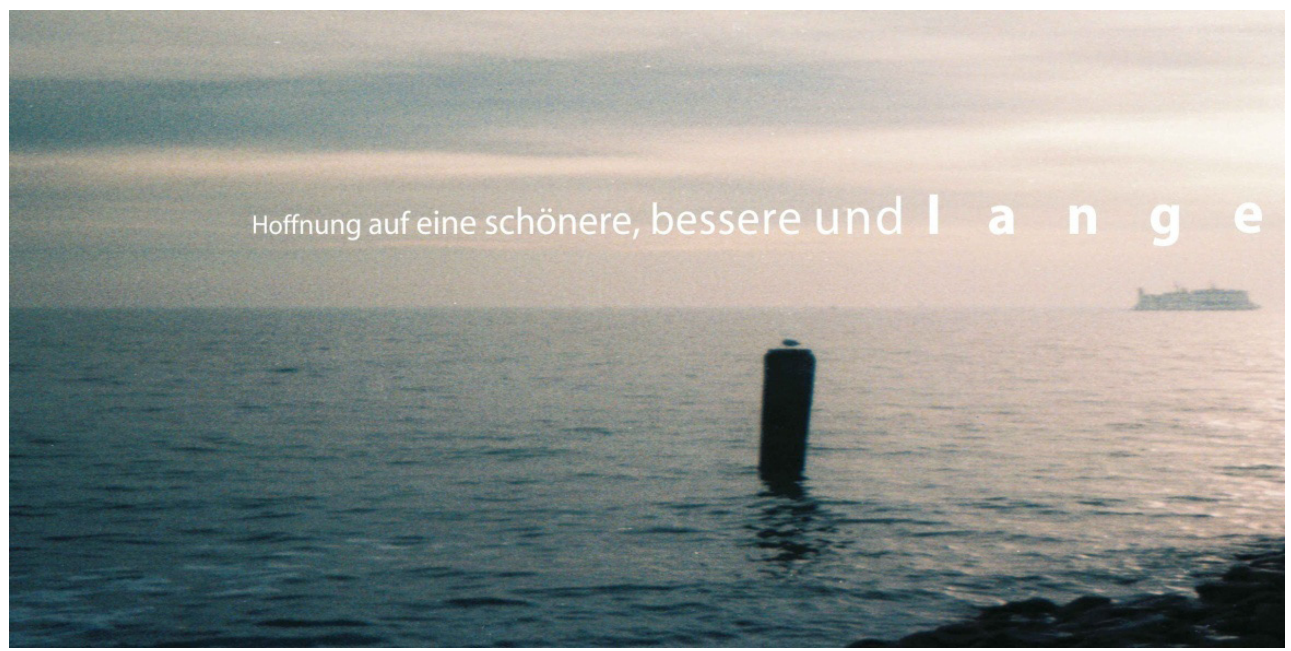

Abb. 19: Foto und Text von Tom (anon.) in der Bearbeitung durch Studierende $(180 \times 80 \mathrm{~cm}$, Druck auf Forex) für die Ausstellung: Bilder und Texte von Rechtsextremen, 2012 (Campus Nord der TU-Braunschweig)

nahmen sie durch die Reduktion auf den Halbsatz die ohnehin vage Richtung (auf eine Zukunft ohne Probleme und Hass). Das Foto-Bild beschnitten sie in der Breite ebenfalls etwa um die Hälfte, ausserdem oben und unten. Das Fragment streckten sie anschliessend über das originale Bildformat $13 \times 9$ hinaus auf $15 \times 8$. Durch den neuen Ausschnitt veränderte sich die atmosphärische Stimmung, denn durch den Zoom-Effekt wurde das neue Bild grobkörniger, damit unklarer. Die nicht definierten Wasser- und Himmelsflächen nehmen durch den Beschnitt im Bild mehr Raum ein, der Horizont wirkt dadurch verwaschener, die Sonne und ihre Spiegelung auf dem Wasser ist rechts nur noch andeutungsweise vorhanden, wodurch das im Originalbild bereits angelegte atmosphärische Rauschen vergrössert wird. Von weiteren Bildelementen wurde das Bild durch den Beschnitt befreit. Das Motiv Schiff ist nun zwar etwas grösser, aber aus dem Zentrum an den rechten Rand gerückt. Dafür rückt nun eine der Buhnen mit dem Umriss des winzigen Vogels ins Zentrum. Dahinein, vor dem Hintergrund einer undefinierbar verschleierten Himmelsfläche, ist das Satzfragment platziert, ebenfalls in der Länge gedehnt, sanft anschwellend mit Betonung auf «l a n g e». Auf geradezu absurde Weise korrespondieren in dieser visuellen Formulierung die vom Sinn weitgehend entleerten Worte auf der Bildebene mit der Leere grosser Bildflächen und der Belanglosigkeit des Fotomotivs Buhne mit Vogelsilhouette.

Die Studierenden machen über das Bild-Text-Arrangement sichtbar und ästhetisch nachvollziehbar, was Toms Fotografien und Kommentare für sie ausdrückten - bis zur Erstarrung gedehnte Zeit, Dinge im Ungewissen lassen, Warten, Perspektivlosigkeit, Unverbindlichkeit, aber auch Hilflosigkeit. 
Ganz anders brachte die Gruppe, die sich mit den Fotos und Texten von Sebastian auseinandergesetzt hatte, seine Äusserungen in einem neuen Arrangement zusammen (Abb. 20). Die klaren Strukturen seiner Bilder und Kommentare wurden von ihnen in einem Rechteck-Arrangement von drei Bildern und einem Teilkommentar aufgenommen, den sie im Bildformat einfügten, klar, schnörkellos, konzentriert auf Kernaussagen. Die ohnehin bildhaft angelegte Teilung der Fotografie mit den zwei Wegen in Abb. 5 wurde von ihnen durch das Trennen in zwei Bilder verstärkt. Damit wurde bei den Betrachtern/-innen von vornherein die Wahrnehmung von und Auseinandersetzung mit zwei Bildern, aber auch der Bezug aufeinander, provoziert.

Diese Tafeln wurden in der hier gezeigten quadratischen Anordnung an die Wand, die anderen Tafeln, auch die Bearbeitung von Toms Foto und Text, in Augenhöhe in die Glasfelder des Hörsaalvorraumes auf dem Campus Nord eingepasst. Für die Dauer der Ausstellung übernahmen die Fensterrahmen der Glaswand des Hörsaalgebäudes die Doppelfunktion von Fenster- und Bilderrahmen, ein gewollter Reflex auf die Doppelfunktion der Fotografie. Zugleich versperrten die Bild-TextArrangements die Blicke der Betrachter/innen nach aussen. Darüber sollten diese angeregt werden, sich sowohl mit der eigenen erfahrenen Blickbeschränkung auseinander zu setzen, während sie sich zugleich mit den Blickhinderungen auseinan-
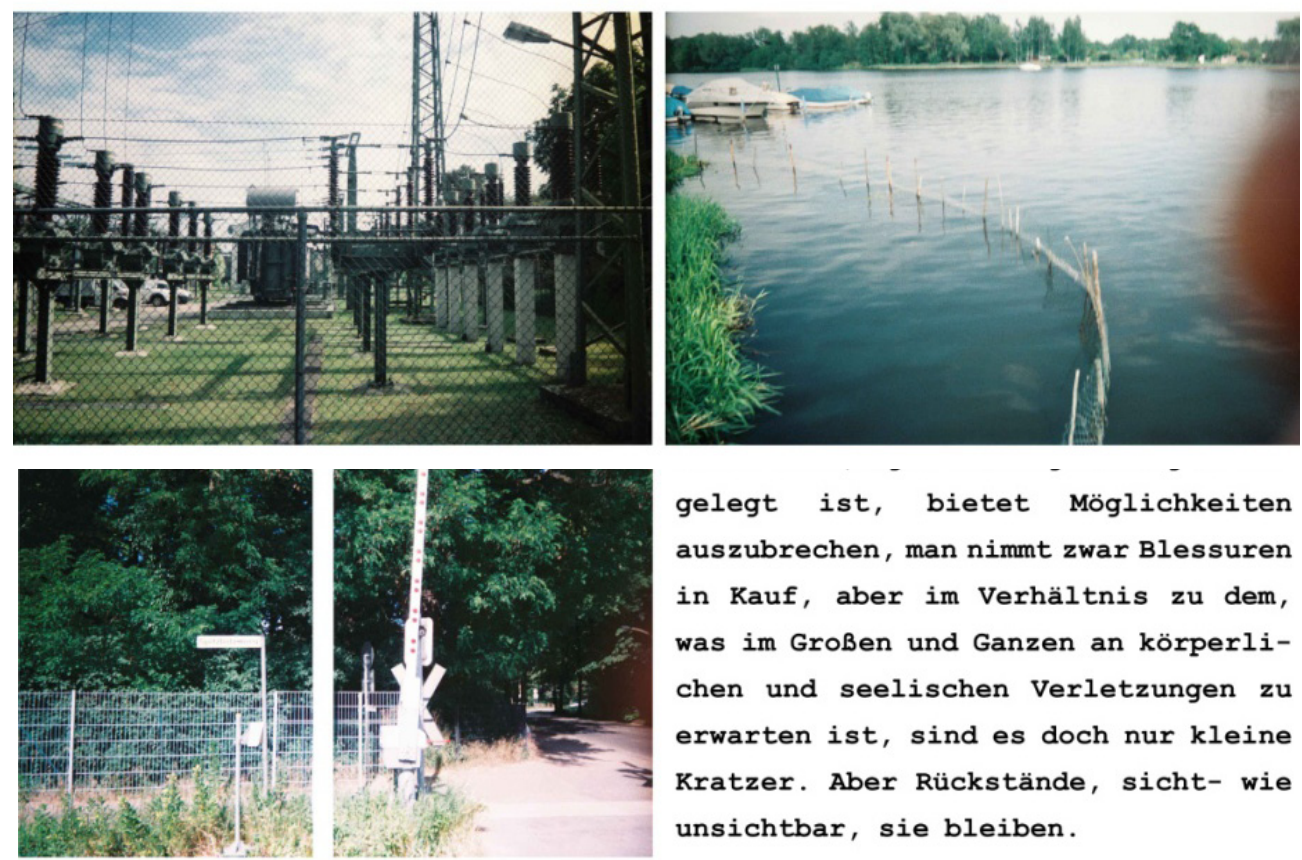

gelegt ist, bietet Möglichkeiten auszubrechen, man nimmt zwar Blessuren in Kauf, aber im Verhältnis zu dem, was im Großen und Ganzen an körperlichen und seelischen Verletzungen zu erwarten ist, sind es doch nur kleine Kratzer. Aber Rückstände, sicht- wie unsichtbar, sie bleiben.

Abb. 20: Fotos und Text von Sebastian (anon.) in der Bearbeitung durch Studierende im Seminar 2012 (2 Platten $180 \times 80 \mathrm{~cm}$, Druck auf Forex) für die Ausstellung: Bilder und Texte von Rechtsextremen. 2012 (Campus Nord der TU-Braunschweig) 
dersetzen mussten, die auf der Ebene der ausgestellten Bild-Text-Arrangements bearbeitet worden waren.

Die Ausstellung wurde am 8. Mai 2012 eröffnet und konnte bis zum März 2013 besichtigt werden, sie fand vor allem unter den Studierenden der TU-Braunschweig grosse Resonanz. Zwischenzeitlich wurde sie vier Wochen in den Räumen der Friedrich-Ebert-Stiftung in Berlin, verbunden mit einer Podiumsdiskussion, gezeigt. Ab April 2013 wird sie in der Geschäftsstelle des Bündnis für Demokratie und Toleranz in Berlin ihren Platz haben.

Die hier vorgestellten Projektphasen und -ebenen, das Projekt «Lebensbilder» in Berlin, initiiert mit Aussteigern/-innen aus der rechtsextremen Szene und die zwei Seminar-Workshops an der TU-Braunschweig, die sich der Analyse und künstlerischen Verarbeitung der Bilder widmeten, haben sich aus inhaltlichen und methodischen Fragestellungen und Problemen entwickelt - das Projekt hatte kein Vorbild und besitzt in dieser Form sicherlich auch keinen Modellcharakter. Gleichwohl lässt sich ein sozial- und medienpädagogischer wie auch bildwissenschaftlicher Ertrag verallgemeinern.

\section{Fazit: Sozial- und medienpädagogischer wie auch bildwissenschaftlicher Ertrag (Fabian Wichmann und Ulrike Pilarczyk)}

In der Projektarbeit «Lebensbilder» zeigte sich, dass mit dem Fotografieren über biografische Bildproduktions- und Reflexionsprozesse in der Ausstiegsbegleitung genuin pädagogische Zielsetzungen verbunden werden können, die die Betroffenen unmittelbar erreichen. Das Projekt hat eindrucksvoll belegt, dass das Fotografieren als Prozess sukzessiver Bilderzeugung Reflexionsprozesse für die (Re-) Identitätsentwicklung und Lebensbewältigung anstossen kann. Für die sozialpädagogische Arbeit war eine solche Intervention nicht nur innovativ, sondern auch produktiv. Die durch das Projekt gewonnenen Erkenntnisse sind in die praktische Ausstiegsarbeit eingeflossen - entweder bei der konsekutiven Auswertung von Ausstiegsverläufen oder in die praktische Ausstiegsbegleitung.

Was die Relevanz der Bildanalysen in den Seminaren für die Auswertung des Fallverlaufs im Rahmen eines Case Managements zeigte, war, dass die Studierenden zunächst ohne Textkontexte und bis zum Schluss ohne Informationen zur Person des Fotografen, die multidimensionalen Problemlagen über eine methodisch geleitete Analyse des visuellen und verbalen Ausdrucks der Fälle herausarbeiten konnten. Die Analysen in den universitären Seminar-Workshops waren in einigen Fällen retrospektiv, in anderen aktuell für die Arbeit des Case Managements von EXIT-Deutschland hilfreich. Bezogen auf die hier dargestellten Fälle kann der Ertrag hier nachträglich festgestellt werden.

Für Tom wurden über die Bild- und Text-Analysen sowohl das Fehlen einer intrinsischen Bedarfslage oder Motivation als auch die externe Motivation seines Aus- 
stieges sichtbar. Deutlicher als im täglichen Umgang wurde seine depressive Störung kenntlich. Hier hat, wie im Text schon erwähnt, die sozialpädagogische Ausstiegsunterstützung ihre Grenzen, es geht dann in solchen Fällen darum, alle verfügbaren Ressourcen zu nutzen, um den erkannten Therapiebedarf angemessen zu vermitteln.

Sebastian lieferte zum Projekt «Lebensbilder» den umfangreichsten Bildkorpus und er fiel auch durch die ausführliche Kommentierung seiner Bilder auf. Hier leitete die Projektarbeit und Analysen ein Umdenken bei den Fallbetreuern ein, von denen er anfänglich als fahrig und unstrukturiert wahrgenommen worden war. Die klare symbolhafte Motivwahl, ein tiefe und bildhafte Sprache zeigten einen kritischen und reflektierten Beobachter gesellschaftlicher Verhältnisse, der darüber hinaus eine ebenso kritische, reflektierte und verantwortungsbewusste Haltung zu seiner eigenen Biografie und sozialen Gewordenheit entwickelt hatte.

Karen befindet sich im akuten Ausstieg. Sie sieht sich permanent mit Unsicherheiten und Problemen konfrontiert, die in einem direkten Zusammenhang mit ihren Aktivitäten innerhalb der rechtsextremen Szene stehen. Sie war mehr als zehn Jahre in unterschiedlichen Kameradschaften und Zusammenschlüssen der rechtsextremen Szene aktiv und ein erster Ausstiegsversuch endete mit einer Tätigkeit als V-Person für den Verfassungsschutz. ${ }^{13}$ Im Bild verhindert der Zaun einen klaren Blick auf das Dahinter, in Karens Lebensrealität verhindert oder erschwert die Vergangenheit sowohl objektiv den Alltag als auch schafft sie subjektive Wahrnehmungs- und Handlungsunsicherheiten, die das Ankommen im Alltag verhindern. Die Rationalisierung und Bewältigung dieser Belastungen (vgl. Greve 2008) stehen im Fokus der Ausstiegsbetreuung. Die Analyse der Bilder machte deutlich, dass sich Karen dieser hemmenden Struktur zumindest auf einer bildhaften Ebene bewusst ist. Zugleich zeigen die Bilder aber auch, dass ihr die Ressourcen fehlen, um sie zu bearbeiten und zu überwinden.

Über die Analyse von Karens Fotografien wurde noch eine andere, über den Einzelfall hinausreichende Erkenntnis gewonnen. Denn im Prozess der Analyse und der Ausstellungsgestaltung wurde ein Kriterium für einen erfolgreichen Ausstieg wichtig, das von der Seite des Case Managements in seiner umfassenden Bedeutung bis dahin noch nicht ausreichend gewürdigt worden war. Denn neben dem Lösen von der Bezugsgruppe, dem Überwinden und Infrage stellen der bisherigen handlungs- und richtungsweisenden Ideologie gibt es mindestens noch eine individuelle Bewältigungsdimension, die einen Ausstieg nachhaltig abschliesst - die Fähigkeit, positiv handlungsfähig sein zu können, die Vergangenheit als nichtänderbaren Teil der Biografie zu akzeptieren und im besten Fall als Erfahrungswert

13 Als V-Personen bezeichnet man Verbindungsperson von Nachrichtendiensten. Sie sind ein nachrichtendienstliches Mittel/Instrument der Informationsgewinnung. Dabei handelt es sich nicht um Mitarbeiter des Nachrichtendienstes, sondern szeneinterne Informanten, die für ihre Tätigkeit entlohnt werden. 
zu begreifen. Die zentralen Aspekte sind dabei die konstruktive Aufarbeitung und Bewältigung biografischer Diskontinuitäten.

Die methodisch geleitete Analyse fotografischer Bilder kann zu einem besseren Verständnis innerer Verarbeitungsprozesse, Diskontinuitäten, individueller Narrative und mentaler Dispositionen führen, was eine gezieltere Planung pädagogischer Intervention ermöglicht. Die Bilder und ihre künstlerischen Bearbeitungen im Rahmen der Ausstellungsarbeit eröffneten auch Möglichkeiten, über Probleme ins Gespräch zu kommen, die bislang gar nicht im Blick waren und wo die Worte fehlen. Diese Chance wurde in der Fallarbeit durch die Mitarbeiter von EXIT-Deutschland kompetent und sensibel genutzt. Da Respekt, Behutsamkeit und Rücksicht unabdingbare Voraussetzungen für erfolgreiche Ausstiegsarbeit sind, haben wir davon abgesehen, Aussteiger/innen unmittelbar mit Ergebnissen der Seminar-Analysen und der Ausstellung zu konfrontieren.

Fotografien, Motive, Stil und die raum-zeitlichen Konstruktionen der Bilder, doch auch die darin angelegte metaphorische Vielfalt drücken Ambivalenz und Komplexität des Prozesses, in dem sich Aussteiger/innen befinden auf eine Weise aus, die den Betroffenen verbalsprachlich nicht zur Verfügung stand. Die Bilder liefern Momentaufnahmen mentaler Dispositionen und verweisen auf Wegzeichen, denn das Fotografieren zwingt auch dort zu bildhafter Konkretion, wo die Sprache Abstrakta bereithält. Dadurch gibt das fotografische Bild auch dort noch Auskunft, wo Sprachlosigkeit herrscht. Das kann denen, die die Bilder erzeugen, helfen, sich selbst und die Situation, in der sie sich befinden, besser zu verstehen, Entscheidungen zu begründen und alternative Handlungs-/Deutungsmuster zu erkennen und zu erschliessen. Das hilft aber auch jenen, die helfen sollen zu verstehen, in welcher Lage sich die zu Betreuenden befinden, was sie hindert, ängstigt und beschränkt, aber auch, ob und was sie hoffen.

Methodisch hat sich das in der seriell-ikonografischen Fotoanalyse angelegte Pendeln zwischen hermeneutischer Arbeit am einzelnen Bild (ikonografisch-ikonologische Einzelbildanalyse) und serieller Prüfung und Analyse bewährt. Zugleich führte die hier erprobte innovative Verschränkung von text- und bildanalytischem Verfahren zu einer neuen Qualität einer sozialwissenschaftlichen Erschliessung komplexer ästhetischer Produkte.

Die bildanalytische Auswertung und die ästhetisch-künstlerische Auseinandersetzung durch Studierende, war nicht nur geeignet, Bild- und Methodenkompetenz zu erwerben, sondern hat hier eine grundsätzliche Sensibilisierung und Verständnis für die komplizierte und widersprüchliche biografische Situationen, wie ihn der Ausstieg aus dem Rechtsextremismus darstellt, geschaffen. ${ }^{14}$

Die Arbeit mit bildbasierten, methodisch kontrollierten Analysen, wozu hier auch die nachfolgende künstlerische Auseinandersetzung gerechnet wurde, ist grund-

14 Das Seminar wurde für den studentischen Lehrpreis LehrLEO 2013 der TU-Braunschweig nominiert. 
sätzlich geeignet, Studierende pädagogischer Fächer - so ist unser Fazit - nachhaltig zu befähigen, biografisch fremde Lebenslagen zu verstehen und pädagogische Interventionen angemessen zu wählen und zu begründen.

\section{Literatur}

Borstel, Dierk. 2011. «Wir hatten auch Spass und haben gelacht ...: Ein- und Ausstiegsprozesse von Männern und Frauen aus der rechtsextremen Szene.»In Rechtsextremismus und Gender, hrsg. v. Ursula Birsl, 297-313. Opladen: Barbara Budrich.

EXIT-Deutschland c/o Zentrum Demokratische Kultur (ZDK). 2011. Lebensbilder: Wege aus dem Extremismus. Ausstellungskatalog, Berlin.

Greve, Werner. 2008. "Bewältigung und Entwicklung.»In Entwicklungspsychologie, hrsg. v. Rolf Oerter u. Leo Montada, 910-926. Weinheim: Beltz.

Hölzle, Christina und Irma Hansen. 2011. Ressourcenorientierte Biografiearbeit: Grundlagen - Zielgruppen - Kreative Methoden. Wiesbaden: VS Verlag.

Möller, Kurt und Nils Schumacher. 2007. Rechte Cliquen: Rechtsextreme Orientierungs- und Szenezusammenhänge - Einstiegs-, Verbleibs- und Ausstiegsprozesse von Skinheads. Wiesbaden: VS Verlag.

Pilarczyk, Ulrike und Ulrike Mietzner. 2005. Das reflektierte Bild: Seriell-ikonografische Fotoanalyse in den Erziehungs- und Sozialwissenschaften. Bad Heilbrunn: Julius Klinkhardt. http://www.pedocs.de/volltexte/2010/2666/

Pilarczyk, Ulrike. 2009. Gemeinschaft in Bildern: Jüdische Jugendbewegung und zionistische Erziehungspraxis in Deutschland und Palästina/Israel. Göttingen: Wallstein.

Pilarczyk, Ulrike. 2006. "Selbstbilder im Vergleich: Junge Fotograf/innen in der DDR und in der Bundesrepublik vor 1989.» In Bildinterpretation und Bildverstehen, hrsg. v. Horst Niesyto u. Winfried Marotzki, 230-253. Wiesbaden: VS Verlag für Sozialwissenschaften.

Pilarczyk, Ulrike. 2004. «Inszenierungen des Selbst in der digitalen Jugendfotografie.» In Imaging: Digitale Fotografie in Schule und Jugendarbeit, hrsg. v. Alfred Holzbrecher u. Jan Schmolling, 79-88. Wiesbaden: VS Verlag für Sozialwissenschaften.

Wagner, Bernd, Fabian Wichmann und Dierk Borstel. 2013. «Modellprojekt der ZDK Gesellschaft Demokratische Kultur gGmbH im Rahmen der Initiative EXITDeutschland am Beispiel des Landkreises Dahme-Spreewald Familien stärken - gegen Extremismus und Gewalt.» Journal EXIT-Deutschland (JEX) 1: S.108153. http://journals.sfu.ca/jed/index.php/jex/article/view/11.

Wouters, Miriam. 2012. «Moving Forwards? Breaking with Right-Wing Extremism in Present Day.» ISRM Working Paper 03/12: http://istramo.com/images/WP_ 3-12_Miriam_Wouters-moving_forwards.pdf. 
Zentrum Demokratische Kultur (ZDK) 2002. « «... dann hab' ich mir das Hitlerbärtchen abrasiert.) Exit - Ausstieg aus der rechtsextremen Szene.» Bulletin. Schriftenreihe des Zentrum Demokratische Kultur, 2/02. Leipzig: Klett Verlag.

Der Abdruck der Fotografien aus dem Projekt «Lebensbilder» und der Ausstellung «Bilder und Texte von Rechtsextremen im Ausstieg" geschieht mit freundlicher Genehmigung von EXIT-Deutschland und der TU-Braunschweig/Fakultät 6. 